\title{
Access to Triplet Excited State in Core-Twisted
}

\section{Perylenediimide}

Kalaivanan Nagarajan, Ajith R. Mallia, V. Sivaranjana Reddy and Mahesh Hariharan*

School of Chemistry, Indian Institute of Science Education and Research Thiruvananthapuram (IISER-

TVM), CET Campus, Sreekaryam, Thiruvananthapuram, Kerala 695 016, Fax: (91)-471-2597427

\section{Supporting Information}

\begin{tabular}{|c|c|}
\hline S. No. & Contents \\
\hline 1 & Materials and methods \\
\hline 2 & Synthesis and Characterization \\
\hline 3 & Scheme S1. Synthesis scheme for PDI- $\mathrm{Br}_{0-4}$. \\
\hline 4 & Table S1. Single crystal analysis of PDI-Br $2-4$. \\
\hline 5 & Table S2. Electrochemical studies of derivatives PDI- $\mathrm{Br}_{0-4}$. \\
\hline 6 & $\begin{array}{l}\text { Table S3. Photophysical properties of derivatives } \mathrm{PDI}^{-\mathrm{Br}_{0-4}} \text { in monomeric (toluene } \\
\text { solution) and polycrystalline state. }\end{array}$ \\
\hline 7 & $\begin{array}{l}\text { Table S4. Oscillator strength for different transitions of PDI and } \mathrm{PDI}_{-} \mathrm{Br}_{4} \text { calculated at } \\
\text { TD-B3LYP/6-311G+ }(\mathrm{d}, \mathrm{p}) \text { level of theory. }\end{array}$ \\
\hline 8 & $\begin{array}{l}\text { Table S5. Low-lying excited singlet }\left(\mathrm{S}_{\mathrm{n}}\right) \text { and triplet }\left(\mathrm{T}_{\mathrm{n}}\right) \text { electronic states of derivatives } \\
\text { PDI-Br } 0 \text {-4 in eV, calculated at TD-B3LYP/6-311G+ }(\mathrm{d}, \mathrm{p}) \text { level of theory. }\end{array}$ \\
\hline 9 & $\begin{array}{l}\text { Table S6. Indicates the efficiency of singlet fission mediated generation of triplet } \\
\text { excited state in representative chromophoric systems. }\end{array}$ \\
\hline 10 & $\begin{array}{l}\text { Table S7. Low-lying excited singlet }\left(\mathrm{S}_{\mathrm{n}}\right) \text { and triplet }\left(\mathrm{T}_{\mathrm{n}}\right) \text { electronic states of PDI-Br } \\
\text { dimer and monomer in eV, calculated at TD-B3LYP/ LANL2dz level of theory } \\
\text { (corresponding oscillator strength is given in the parenthesis). }\end{array}$ \\
\hline 11 & Figure S1. ${ }^{1} \mathrm{H}-\mathrm{NMR}$ spectra of derivatives $\mathrm{PDI}-\mathrm{Br}_{0-4}$ in $\mathrm{CDCl}_{3}$. \\
\hline 12 & $\begin{array}{l}\text { Figure S2. a) Overlapped aromatic area and b) total aromatic surface area between the } \\
\text { vicinal perylenediimide moieties of PDI-Br }{ }_{4} \text { obtained from the crystal packing; c) and } \\
\text { d) short contact between the vicinal PDI units in } \mathrm{PDI}-\mathrm{Br}_{3} \text { and } \mathrm{PDI}-\mathrm{Br}_{4} \text {, respectively. }\end{array}$ \\
\hline 13 & $\begin{array}{l}\text { Figure S3. Cyclic voltammogram of } \mathrm{PDI}-\mathrm{Br}_{0-4} \text { in dichloromethane with respect to } \\
\mathrm{Ag} / \mathrm{AgCl} \text { reference electrode. }\end{array}$ \\
\hline 14 & $\begin{array}{l}\text { Figure S4. Representative frontier molecular orbital diagrams of the model derivative } \\
\text { PDI and the PDI-Br } 4 \text { calculated at TD-B3LYP/6-311G+ }(d, p) \text { level of theory. }\end{array}$ \\
\hline 15 & $\begin{array}{l}\text { Figure S5. Picosecond time-resolved fluorescence measurements of PDI- } \mathrm{Br}_{0-4} \text { in } \\
\text { toluene (excitation wavelength: } 439 \mathrm{~nm} \text { ) monitored at the respective emission maxima. }\end{array}$ \\
\hline
\end{tabular}

Page

S3

S9

S9

S11

S12

S12

S12

S13

S13

S14

S15

S15

S16

S17

S18 
Figure S6. Femtosecond transient absorption spectrum of a) PDI; c) PDI-Br; e) PDI$\mathrm{Br}_{2}$; g) PDI-Br 3 ; in toluene; excited at $\left.\left.400 \mathrm{~nm} \mathrm{~b}\right), \mathrm{d}\right), \mathrm{f}$ ) and h) are corresponding SVD.

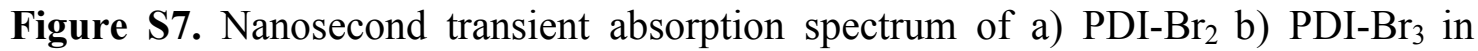
toluene; excited at $532 \mathrm{~nm}$; c) near-quadratic increase in the core-twist angle, triplet quantum yield in toluene solution and solid state fluorescence quantum yield of PDI$\mathrm{Br}_{0-4}$ with the linear increase in number of bromine atoms.

Figure S8. Sandros relation plot for $\mathrm{PDI}_{-} \mathrm{Br}_{4}$ calculated from nanosecond transient absorption measurements.

Figure S9. a) Energy level diagram for the PDI and $\mathrm{PDI}-\mathrm{Br}_{1-4}$ derivatives calculated at TD-B3LYP/6-311G+ (d, p) level of theory b) energy difference between various excited states.

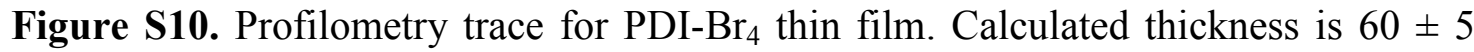
nm.

Figure S11. PXRD diffractogram of PDI-Br 4 thinfilm (black trace) overlaid with the diffractogram calculated from the single crystal structure of $\mathrm{PDI}_{-} \mathrm{Br}_{4}$ (red trace).

S20

Figure S12. Ground state raman spectra of $\mathrm{PDI}_{-\mathrm{Br}_{4}}$ in toluene solution (black trace)

22 and polycrystalline thinfilm state (red trace) on excitation with $480 \mathrm{~nm}$ laser (*denotes toluene absorption band).

Figure S13. Picosecond time-resolved fluorescence measurements of $\mathrm{PDI}_{-\mathrm{Br}_{3-4}}$ in

23 toluene and in thin-film state on exciting at $439 \mathrm{~nm}$ and monitored at the respective emission maxima.

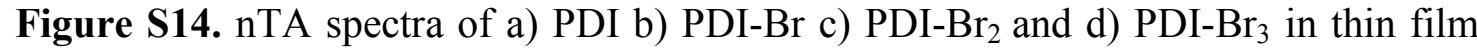
state.

Figure S15. Excitation density dependence of the ground state depletion of polycrystalline $\mathrm{PDI}_{-\mathrm{Br}_{4}}$ on excitation at $532 \mathrm{~nm}$ (probed at $550 \mathrm{~nm}$ ).

Figure S16. a) $\mathrm{PDI}_{-} \mathrm{Br}_{4}$ triplet absorption spectrum derived from the nTA spectrum at $50 \mathrm{~ns}$ following $7 \mathrm{~ns}, 532 \mathrm{~nm}$ laser pulse and the ground state (GS) absorption spectrum in polycrystalline thin film. ${ }^{1}$ b) the absorbance required to completely account for the ground state bleach is roughly 0.0782 that predicted by the calculation. The observed bleach is 0.0616 . So the calculated triplet yield $=0.0616 / 0.0782=0.788(79 \pm 5 \%)$.

Figure S17. a) Quantum yield of triplet generation in monomeric and polycrystalline thin film of PDI-Br 4 , on exciting at 355 and $532 \mathrm{~nm}$.

Figure S18. Show a) ground state absorption spectra; b) relative optical density and PMMA blend thin film. 


\section{Materials and Methods:}

All chemicals were obtained from commercial suppliers and used as received without further purification. All reactions were carried out in oven-dried glassware prior to use and wherever necessary. All reactions were performed under dry nitrogen in dried, anhydrous solvents using standard gastight syringes, cannulae, and septa. Solvents were dried and distilled by standard procedures. TLC analyses were performed on precoated aluminum plates of silica gel 60 F254 plates $(0.25 \mathrm{~mm}$, Merck) and developed TLC plates were visualized under short and long wavelength UV lamps. Column chromatography was performed using silica gel of 200-400 mesh employing a solvent polarity correlated with the TLC mobility observed for the substance of interest. Yields refer to chromatographically and spectroscopically homogenous substances. Melting points were obtained using a capillary melting point apparatus and are uncorrected. IR spectra were recorded on a Shimadzu IRPrestige-21 FT-IR spectrometer as neat thin films between $\mathrm{NaCl}$ plates in case of liquids and as $\mathrm{KBr}$ pellets in the case of solids. ${ }^{1} \mathrm{H}$ and ${ }^{13} \mathrm{C}$ NMR spectra were measured on a $500 \mathrm{MHz}$ and $125 \mathrm{MHz}$ Bruker advanced DPX spectrometer respectively. Internal standard used for ${ }^{1} \mathrm{H}$ and ${ }^{13} \mathrm{C}$ NMR is $1,1,1,1-$ tetramethyl silane (TMS). All CHN analyses were carried out on an Elementar vario MICRO cube Elemental Analyzer. All values recorded in elemental analyses are given in percentages. High Resolution Mass Spectra (HRMS) were recorded on a Agilent 6538 Ultra High Definition (UHD) Accurate-Mass Q-TOF-LC/MS system using either atmospheric pressure chemical ionization (APCI) or electrospray ionization (ESI) mode. Thin film samples were prepared by spin-coating saturated chloroform solution of PDI-Br${ }_{0-4}$ on quartz plate.

\section{X-ray Crystallography:}

High-quality specimens of appropriate dimensions were selected for the X-ray diffraction experiments. Crystallographic data collected are presented in the supplementary information. Single crystals were mounted using oil (Infineum V8512) on a glass fibre. All measurements were made on a $\mathrm{CCD}$ area detector with graphite monochromated Mo K $\alpha$ radiation. The data was collected using Bruker APEXII detector and processed using APEX2 from Bruker. All structures were solved by direct methods and expanded using Fourier techniques. The non-hydrogen atoms were refined anisotropically. Hydrogen atoms were included in idealized positions, but not refined. Their positions were constrained relative to their parent atom using the appropriate HFIX command in SHELXL-97. The full validation 
of CIFs and structure factors of PDI- $\mathrm{Br}_{2-4}$ were performed using the CheckCIF utility and found to be free of major alert level. 3D structure visualization and the exploration of the crystal packing of $\mathrm{PDI}_{-} \mathrm{Br}_{2}$ 4 were carried out using Mercury 3.1. Percentage (\%) overlap was calculated from area of overlapped moieties of perylenediimide aromatic rings in the crystal structures. Percentage of overlapped aromatic surface area to total aromatic surface area gives the $\%$ overlap. $^{2}$

\section{Spectral Measurements:}

Absorption spectra were recorded in Shimadzu UV-3600 UV-Vis-NIR while emission (fluorescence/phosphorescence) and excitation spectra were performed in Horiba Jobin Yvon Fluorolog spectrometer. All spectroscopic experiments were performed by using standard quartz cuvettes of path length $1 \mathrm{~cm}$ for solution in dried and distilled solvents. The solution state fluorescence quantum yields were determined by using optically matched solutions. Fluorescein dissolved in ethanol $\left(\Phi_{\mathrm{fl}}=79 \%\right)^{3}$ was used as the standard. The fluorescence quantum yield was calculated as follows,

$$
\Phi=\Phi_{R} \times \frac{I}{I_{R}} \times \frac{A_{R}}{A} \times \frac{\eta^{2}}{\eta_{R}^{2}}
$$

Phosphorescence measurement was performed in Quartz tube in chloroform/ethyl iodide solid glasses at 77K in Horiba Jobin Yvon Fluorolog spectrometer. During the measurement, time per flash was 61 and delay after the flash was $0.05 \mathrm{~ms}$.

The solid state quantum yield of model derivative PDI and PDI-Br $\mathrm{P}_{1-4}$ were measured using an integrating sphere for which the accuracy was verified using tris(8-hydroxyquinolinate)aluminium $\left(\mathrm{Alq}_{3}\right)$ as a standard and is determined to be $37 \pm 4 \%$ (reported quantum yield $\left.\Phi_{\mathrm{fl}}=40 \%\right)^{4}$

Fluorescence decay measurements were carried out in an IBH picosecond single photon counting system. The excitation laser used was $439 \mathrm{~nm}$ with a pulse width of less than $100 \mathrm{ps}$. The fluorescence decay profiles were deconvoluted using IBH data station software version 2.1 and fitted, minimizing the $\chi^{2}$ values of the fit to $1 \pm 0.05$.

\section{Femtosecond Transient Absorption Measurement (fTA):}

Spectra-physics Tsunami Oscillator ( $80 \mathrm{MHz}, 800 \mathrm{~nm}$ ) was used as seed for a Spectra-Physics Spitfire Regenerative amplifier $(1 \mathrm{KHz}, 4 \mathrm{~mJ})$. A fraction of the amplified output was used to generate 
$400 \mathrm{~nm}$ pump pulse. Residual $800 \mathrm{~nm}$ pulse was sent through a delay line inside an ExciPro pump-probe spectrometer from CDP Systems. A rotating $\mathrm{CaF}_{2}$ plate $(2 \mathrm{~mm}$ thickness) was used to generate continuum of white light from the delayed $800 \mathrm{~nm}$ pulses. The continuum of white light was split into two and the streams were used as probe and reference pulses. Transient absorption spectra were recorded using a dual diode array detector with a $200 \mathrm{~nm}$ detection window. Sample solutions were prepared in a rotating sample cell with $400 \mu \mathrm{m}$ path length. IRF was determined by solvent $(10 \%$ Benzene in Methanol) two photon absorption and was found to be approximately $130 \mathrm{fs}$ at about $530 \mathrm{~nm}$. Energy per pulse incident on the sample is attenuated employing $80 \%$ neutral density filter when required. Toluene solution of the derivatives PDI-Br${ }_{1-4}$ and the model derivative PDI were pumped with $400 \mathrm{~nm}$ laser and probed by the white light. ${ }^{5}$

\section{Nanosecond Transient Absorption Measurement (nTA):}

Laser flash photolysis experiments of the argon purged solutions were carried out in an Applied Photophysics Model LKS-60 laser kinetic spectrometer using the second and third harmonic $(355 \mathrm{~nm}$ and $532 \mathrm{~nm}$, pulse duration $\approx 7 \mathrm{~ns}$ ) of a Quanta Ray INDI-40-10 series pulsed Nd:YAG laser. Triplet states of the PDI- $\mathrm{Br}_{0-4}$ in toluene were confirmed using the measurement of oxygen purged solutions through nanosecond flash photolysis studies. Triplet quantum yields ${ }^{6}$ upon direct photoexcitation (532 $\mathrm{nm})$ were determined by using $[\mathrm{Ru}(\mathrm{bpy})] \mathrm{C} 1_{2}$ in methanol as standard $\left(\Phi_{\mathrm{T}}=100 \%\right)$, with nonsaturating laser intensities. Equal volume of $0.2 \mathrm{mM}$ solution of $\beta$-carotene was added to optically matched solutions of reference and the sample. The equation for the triplet quantum yield is given by,

$$
\Phi_{T}^{S}=\Phi_{T}^{R e f} \times \frac{\Delta A^{S}}{\Delta A^{R e f}} \times \frac{k_{o b s}^{S}}{k_{o b s}^{S}-k_{0}^{S}} \times \frac{k_{o b s}^{R e f}-k_{0}^{R e f}}{k_{o b s}^{R e f}}
$$

Where, $\Phi_{T}^{S}$ and $\Phi_{T}^{R e f}$ denote the triplet quantum yield of the sample and reference respectively; $\Delta A^{S}$ and $\Delta A^{R e f}$ are transient absorption intensity of $\beta$-carotene in sample and reference respectively; $k_{o b s}^{S}$ and $k_{0}^{S}$ are decay rate of sample transient species before and after the addition of $\beta$-carotene. $k_{o b s}^{R e f}$ and $k_{0}^{R e f}$ are decay rate of reference transient species before and after the addition of $\beta$-carotene.

The triplet energy of the PDI- $\mathrm{Br}_{4}$ was estimated from rate constants for triplet energy transfer by using the Sandros relation ${ }^{7}$ given in the following equation, 


$$
k_{Q}=k_{\text {diff }}\left(1 /\left(1+\exp \left(-\frac{\Delta E_{T}}{k T}\right)\right)\right.
$$

where $k_{\mathrm{Q}}$ is the bimolecular rate constant for energy transfer, $k_{\text {diff }}$ is the diffusion-limited value of $k_{\mathrm{Q}}$, and $\Delta \mathrm{E}_{\mathrm{T}}$ is the triplet energy difference between the donor and acceptor. The values of $k_{\mathrm{Q}}$ were obtained from the slopes of the pseudo-first-order decay rate constant $\left(k_{\mathrm{obs}}\right)$ of the donor triplet versus the concentration of acceptor according to the following equation

$$
k_{o b s}=k_{0}+k_{Q}(\text { acceptor })
$$

where $k_{o}$ is the first-order rate constant for decay of the donor triplet in the absence of acceptor. $\beta$ carotene and $\mathrm{Ru}(\mathrm{bpy})_{3} \mathrm{Cl}_{2}$ were used as triplet energy acceptor whereas anthracene, phenanthrene and coronene were used as triplet energy acceptor in the study with $\mathrm{PDI}-\mathrm{Br}_{4}$.

\section{Relative Triplet Yield Calculation in Solution:}

Triplet quantum yield on exciting at $532 \mathrm{~nm}=18 \pm 1 \%$

Transient absorption on exciting at $355 \mathrm{~nm}=0.03$

Transient absorption on exciting at $532 \mathrm{~nm}=0.1444$

Ground state absorption at $355 \mathrm{~nm}=0.0199$

Ground state absorption at $532 \mathrm{~nm}=0.21$

$$
\begin{gathered}
\varphi_{T(355 \mathrm{~nm})}=\varphi_{T(532 \mathrm{~nm})} \times \frac{\Delta A_{355 \mathrm{~nm}}}{\Delta A_{532 \mathrm{~nm}}} \times \frac{A_{532 \mathrm{~nm}}}{A_{355 \mathrm{~nm}}} \\
\varphi_{T(355 \mathrm{~nm})}=((18 \pm 1) \times 0.03 \times 0.1444) /(0.21 \times 0.0199)=19 \pm 1 \%
\end{gathered}
$$

\section{Triplet Yield Calculation for Singlet Fission:}

Film thickness : $60 \pm 5 \mathrm{~nm}$,

Spot size : $0.785 \mathrm{~cm}^{2}$

Unit cell volume: $1583 \AA^{3}$

Formula units/cell: 2

Excitation wavelength: $532 \mathrm{~nm}$

Excitation pulse energy: $2 \pm 0.1 \mathrm{~mJ}$

Absorbance at $532 \mathrm{~nm}$ : 0.195 \pm 0.004

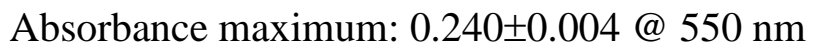




$$
\begin{gathered}
\xi=\frac{E \cdot \lambda \cdot K \cdot\left(1-10^{-A}\right)}{l \cdot a} \\
\xi=\frac{0.002 \mathrm{~J} \cdot 532 \mathrm{~nm} \cdot 5.034 \times 10^{15} \mathrm{~J}^{-1} \mathrm{~nm}^{-1} \cdot\left(1-10^{-0.195}\right)}{60 \times 10^{-7} \cdot 0.785 \mathrm{~cm}^{2}} \\
\xi=(4.1132 \pm 0.05) \times 10^{20}
\end{gathered}
$$

Number of density $=2$ molecules $/ 1.583 \times 10^{-21} \mathrm{~cm}^{3}=1.263 \times 10^{21}$

$\xi /$ no. of density $=0.3256 \pm 0.01$

Expected bleach $=-(0.3256 \pm 0.01) \times(0.240 \pm 0.004)=-0.0782 \pm 0.002$

Observed bleach $=0.0616 \pm 0.004$

Number of ground state molecule lost $=0.0616 / 0.0782=79 \pm 5 \%$

\section{Relative Triplet Yield Calculation in polycrystalline Thin Film:}

Triplet quantum yield on exciting at $532 \mathrm{~nm}=79 \pm 5 \%$

Transient absorption on exciting at $355 \mathrm{~nm}=0.01$

Transient absorption on exciting at $532 \mathrm{~nm}=0.028$

Ground state absorption at $355 \mathrm{~nm}=0.0371$

Ground state absorption at $532 \mathrm{~nm}=0.1386$

$$
\begin{gathered}
\varphi_{T(355 \mathrm{~nm})}=\varphi_{T(532 \mathrm{~nm})} \times \frac{\Delta A_{355 \mathrm{~nm}}}{\Delta A_{532 \mathrm{~nm}}} \times \frac{A_{532 \mathrm{~nm}}}{A_{355 \mathrm{~nm}}} \\
\varphi_{T(355 \mathrm{~nm})}=((79 \pm 5) \times 0.01 \times 0.1386) /(0.028 \times 0.0371)=105 \pm 5 \%
\end{gathered}
$$

\section{Computational Methods:}

Ground-state optimised structure and harmonic oscillator frequencies were computed using density functional theory (DFT) at the Becke's three parameter functional in combination with the LeeYang-Parr correlation functional (B3LYP) and 6-31+G(d,p) basis set. Vertical excitation energies and oscillator strengths were calculated employing time dependent DFT (TD-DFT) at the B3LYP/6$311+\mathrm{G}(\mathrm{d}, \mathrm{p})$ level of theory. Vertical excitation energy and oscillator strength for the slip stacked dimer were calculated from TD-DFT at the B3LYP/LANL2dz level of theory. All computations were performed with the Gaussian 09 program suite. ${ }^{8}$ 


\section{Quantum Theory of Atoms in Molecules (QTAIM):}

The wave function for the derivative $\mathrm{PDI}-\mathrm{Br}_{3-4}$ are obtained employing the geometries taken from the crystal structure using Gaussian set of codes at B3LYP/6-31+G(d,p) level. Quantum theory of atoms in molecules (QTAIM) analysis aims at understanding the description of interatomic interaction in the single crystal X-ray structure. A bond is defined along the bond line between two nuclei, called a bond path, along which electron density is concentrated. In a three dimensional space there are four types of critical points, corresponding to non-degenerate points: termed $(3,-3),(3,-1),(3,+1)$ and $(3$, $+3)$. The $(3,-3)$ and $(3,+3)$ types represent a maximum (which corresponds to a nuclear position) and a minimum, respectively. While $(3,-1)$ and $(3,+1)$ types represent saddle points called bond critical points and the ring critical points, respectively. The bond critical point (BCP) is a point along the bond path at the interatomic surface, where the shared electron density reaches a minimum. The physical characteristics of the BCPs [the electron density at BCP, $\rho(\mathrm{rBCP})$, and its Laplacian, $\nabla^{2} \rho(\mathrm{rBCP})$ ] reveal the approximate measure of the amount of electron density built up in the bonding region and as such could be taken as characteristic of the bond. When $\nabla^{2} \rho(\mathrm{rBCP})<0$ and is large in magnitude, $\rho(\mathrm{rBCP})$ is also large which means that there is a concentration of electronic charge in the internuclear region. This is also an indication of a sharing of electronic charge between both nuclei that defines the covalent (polar) bond. When $\nabla^{2} \rho(\mathrm{rBCP})<0$ there is a depletion of electronic charge in the internuclear region. Using the AIM 2000 software package, the electron density was integrated over atomic basins according to the quantum theory of atoms in molecules using PROAIM, and thus the BCP data and the molecular graphs were obtained. 


\section{Synthesis and Characterisation:}

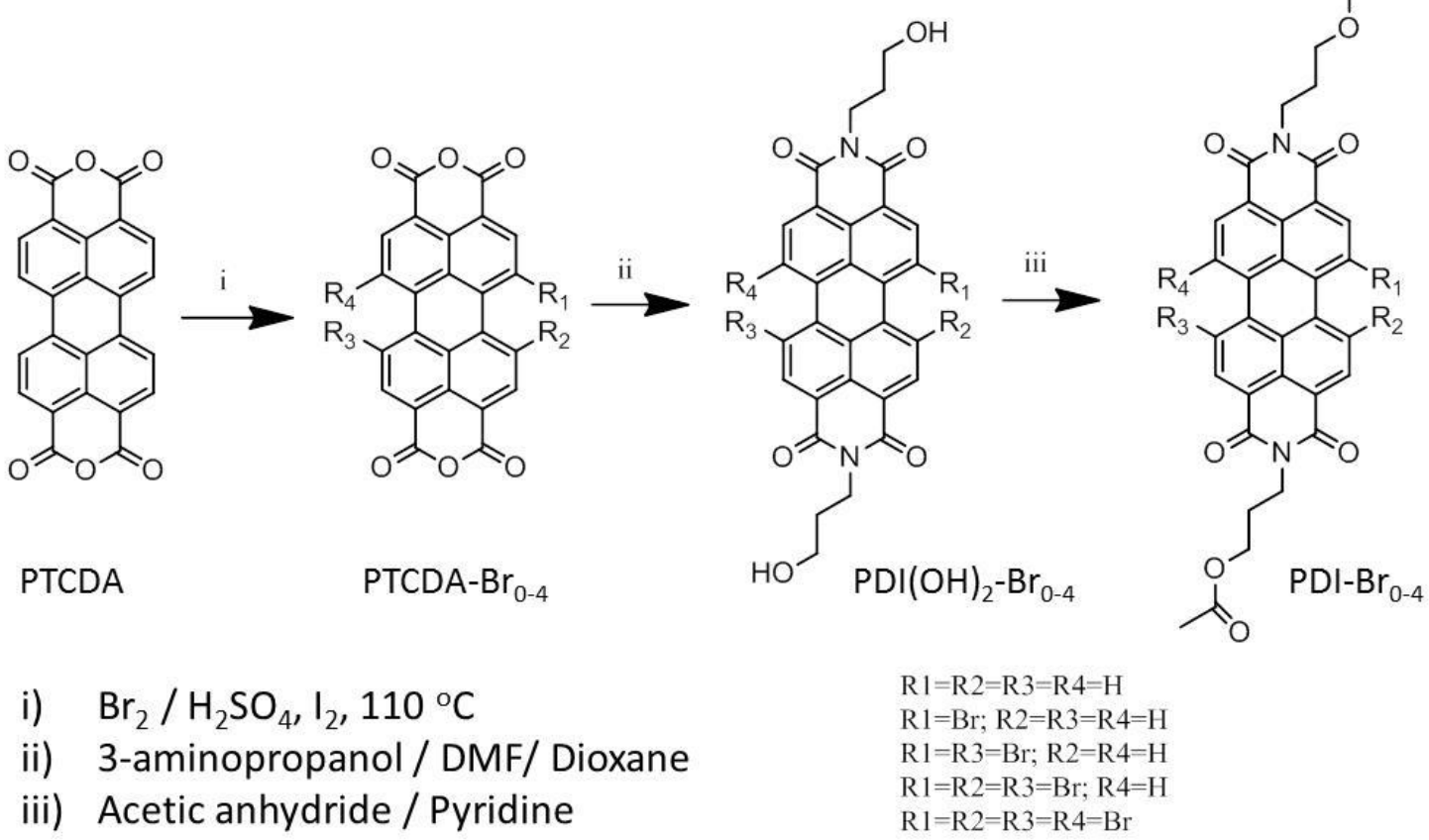

Scheme S1. Synthesis scheme for PDI-Br $\mathrm{B}_{0-4}$.

Synthesis of PTCDA-Br ${ }_{1-4}$ : 7.5 g of perylene-3,4,9,10-tetracarboxylic dianhydride (PTCDA) was stirred with $80 \mathrm{~mL}$ of concentrated sulphuric acid for 4 hours. $500 \mathrm{mg}$ of elemental iodine was added and the reaction mixture was heated to $110^{\circ} \mathrm{C} .1 / 2 / 3$ or 4 equivalents of elemental bromine was added drop wise to synthesise mono, di, tri and tetra bromo PTCDA. The reaction mixture was refluxed for 24 hours. After 24 hours, the product was precipitated by pouring the reaction mixture into $200 \mathrm{~mL}$ of ice water. The product (PTCDA-Br $\mathrm{P}_{1-4}$ ) was filtered and dried in hot air oven. Owing to the insolubility of the compounds, they were taken for next step of synthesis without any characterisation.

Synthesis of PDI-Br $\mathbf{r}_{0-4}: 7.5 \mathrm{~g}$ of PTCDA/PTCDA-Br $\mathrm{B}_{1-4}$ was taken in $75 \mathrm{~mL}$ of $\mathrm{N}, \mathrm{N}$-dimethylacetamide and $75 \mathrm{~mL}$ of 1,4-dioxane and stirred for 30 minutes. 2.2 equivalents of 3-aminopropanol was added dropwise into the reaction mixture at $80^{\circ} \mathrm{C}$. The reaction mixture was refluxed at $110{ }^{\circ} \mathrm{C}$ for 2 hours. After 2 hours, the reaction mixture was cooled to room temperature and poured into $200 \mathrm{~mL}$ of ice water. Precipitated product was filtered and dried in hot air oven. The imidisation was confirmed by IR spectroscopic technique by monitoring the $\mathrm{C}=\mathrm{O}$ stretching. $\mathrm{IR}\left(\mathrm{KBr}, \mathrm{cm}^{-1}\right)$ : 3397, 1694, and 1651 . The dried product was acetylated by treating with excess equivalent of acetic anhydride in dried pyridine. 
The final products $\mathrm{PDI}-\mathrm{Br}_{0-4}$ were purified by column chromatography. All the products have been characterised by spectroscopic and analytical techniques.

PDI (yield = 50 \%). M.p. > $300{ }^{\circ} \mathrm{C} .{ }^{1} \mathrm{H}$ NMR [ $\left.500 \mathrm{MHz}, \mathrm{CDCl}_{3}, \delta\right]: 8.58(\mathrm{~d}, \mathrm{~J}=7.50 \mathrm{~Hz}, 4 \mathrm{H}), 8.49$ (d, J $=7.50 \mathrm{~Hz}, 4 \mathrm{H}), 4.28(\mathrm{t}, \mathrm{J}=6.5 \mathrm{~Hz}, 4 \mathrm{H}), 4.16(\mathrm{t}, \mathrm{J}=6.15 \mathrm{~Hz}, 4 \mathrm{H}), 2.08(\mathrm{~m}, 4 \mathrm{H}), 1.99(\mathrm{~s}, 6 \mathrm{H}) .{ }^{13} \mathrm{C} \mathrm{NMR}$ $\left[125 \mathrm{MHz}, \mathrm{CDCl}_{3}, \delta\right]: 170.22,159.51,135.84,131.20,131.05,129.42,126.36,124.65,60.73,39.34$, 26.27, 20.92. IR $\left(\mathrm{KBr}, \mathrm{cm}^{-1}\right)$ : 1732, 1694 and 1657. Elemental analysis: calcd. value for $\mathrm{C}_{34} \mathrm{H}_{26} \mathrm{~N}_{2} \mathrm{O}_{8}$ : $69.15 \% \mathrm{C}, 4.44 \% \mathrm{H}$ and $4.74 \% \mathrm{~N}$; found: $69.05 \% \mathrm{C}, 4.50 \% \mathrm{H}$ and $4.65 \% \mathrm{~N}$. HRMS (ESI) $\mathrm{m} / \mathrm{z}$ calculated for $\mathrm{C}_{34} \mathrm{H}_{26} \mathrm{~N}_{2} \mathrm{O}_{8}[\mathrm{M}]^{+}: 590.5788$, found: 590.6107 .

PDI-Br (yield = 30 \%). M.p. > $300{ }^{\circ} \mathrm{C} .{ }^{1} \mathrm{H}$ NMR $\left[500 \mathrm{MHz}, \mathrm{CDCl}_{3}, \delta\right]: 9.69(\mathrm{~d}, \mathrm{~J}=8.00 \mathrm{~Hz}, 1 \mathrm{H}), 8.81$ (s, 1H), $8.60(\mathrm{~m}, 3 \mathrm{H}), 8.48$ (d, J= $8.00 \mathrm{~Hz}, 2 \mathrm{H}), 4.27$ (m, 4H), 4.15 (m, 4H), 2.08 (m, 4H), 1.99 (s, 6H). ${ }^{13} \mathrm{C}$ NMR $\left[125 \mathrm{MHz}, \mathrm{CDCl}_{3}, \delta\right]: 170.48,159.67,140.45,135.70,133.20,131.86,131.24,131.08,129.47$, $128.17,127.33,126.82,124.71,124.04,60.85,39.42,26.43,20.84 . \mathrm{IR}\left(\mathrm{KBr}, \mathrm{cm}^{-1}\right)$ : 1735,1693 and 1653. Elemental analysis: calcd. value for $\mathrm{C}_{34} \mathrm{H}_{25} \mathrm{BrN}_{2} \mathrm{O}_{8}: 61.00 \% \mathrm{C}, 3.76 \% \mathrm{H}$ and $4.18 \% \mathrm{~N}$; found: $61.15 \% \mathrm{C}, 3.68 \% \mathrm{H}$ and $4.14 \% \mathrm{~N}$. HRMS (ESI) $\mathrm{m} / \mathrm{z}$ calculated for $\mathrm{C}_{34} \mathrm{H}_{25} \mathrm{BrN}_{2} \mathrm{O}_{8}[\mathrm{M}]^{+}: 669.4749$, found: 669.5005 .

PDI-Br 2 (yield = 70 \%). M.p. > $300{ }^{\circ} \mathrm{C} .{ }^{1} \mathrm{H}$ NMR $\left[500 \mathrm{MHz}, \mathrm{CDCl}_{3}, \delta\right]: 9.41(\mathrm{~d}, \mathrm{~J}=8.20 \mathrm{~Hz}, 2 \mathrm{H}), 8.84$ (s, 2H), $8.62(\mathrm{~d}, \mathrm{~J}=8.20 \mathrm{~Hz}, 2 \mathrm{H}), 4.25(\mathrm{t}, \mathrm{J}=7.1 \mathrm{~Hz}, 4 \mathrm{H}), 4.11(\mathrm{t}, \mathrm{J}=6.10 \mathrm{~Hz}, 4 \mathrm{H}), 2.04(\mathrm{~m}, 4 \mathrm{H}), 1.95$ (s, 6H). ${ }^{13} \mathrm{C}$ NMR $\left[125 \mathrm{MHz}, \mathrm{CDCl}_{3}, \delta\right]: 171.09,162.40,138.11,133.16,132.98,130.16,129.31$, $128.61,126.98,123.04,122.62,120.90,62.20,37.86,27.34,20.95 . \mathrm{IR}\left(\mathrm{KBr}, \mathrm{cm}^{-1}\right): 1733,1698$ and 1670. Elemental analysis: calcd. value for $\mathrm{C}_{34} \mathrm{H}_{24} \mathrm{Br}_{2} \mathrm{~N}_{2} \mathrm{O}_{8}: 54.57 \% \mathrm{C}, 3.23 \% \mathrm{H}$ and $3.74 \% \mathrm{~N}$; found: $54.68 \% \mathrm{C}, 3.29 \% \mathrm{H}$ and $3.61 \% \mathrm{~N}$. HRMS (ESI) $\mathrm{m} / \mathrm{z}$ calculated for $\mathrm{C}_{34} \mathrm{H}_{24} \mathrm{Br}_{2} \mathrm{~N}_{2} \mathrm{O}_{8}$ [M] $]^{+}: 748.3710$, found: 748.3754 .

PDI-Br $_{3}$ (yield = 60 \%). M.p. $>300{ }^{\circ} \mathrm{C} .{ }^{1} \mathrm{H}$ NMR [500 MHz, $\left.\mathrm{CDCl}_{3}, \delta\right]: 9.37(\mathrm{~d}, \mathrm{~J}=8.05 \mathrm{~Hz}, 1 \mathrm{H}), 8.85$ (s, 1H), $8.75(\mathrm{~d}, \mathrm{~J}=1.50 \mathrm{~Hz}, 2 \mathrm{H}), 8.64(\mathrm{~d}, \mathrm{~J}=8.05 \mathrm{~Hz}, 1 \mathrm{H}), 4.26(\mathrm{~m}, 4 \mathrm{H}), 4.13(\mathrm{~m}, 4 \mathrm{H}), 2.06(\mathrm{~m}, 4 \mathrm{H})$, 1.99 (s, 3H), 1.98 (s, 3H). ${ }^{13} \mathrm{C}$ NMR [125 MHz, $\left.\mathrm{CDCl}_{3}, \delta\right]: 170.05,159.14,136.27,132.67,130.81$, $130.25,129.04,128.55,128.32,127.50,124.24,123.57,60.44,39.13,25.78,20.46 . \mathrm{IR}\left(\mathrm{KBr}, \mathrm{cm}^{-1}\right)$ : 1738, 1701 and 1662. Elemental analysis: calcd. value for $\mathrm{C}_{34} \mathrm{H}_{23} \mathrm{Br}_{3} \mathrm{~N}_{2} \mathrm{O}_{8}: 49.36 \% \mathrm{C}, 2.80 \% \mathrm{H}$ and 
$3.39 \% \mathrm{~N}$; found: $49.18 \% \mathrm{C}, 2.92 \% \mathrm{H}$ and $3.30 \% \mathrm{~N}$. HRMS (ESI) m/z calculated for $\mathrm{C}_{34} \mathrm{H}_{23} \mathrm{Br}_{3} \mathrm{~N}_{2} \mathrm{O}_{8}$ $[\mathrm{M}]^{+}:$827.2610, found: 827.2967.

PDI-Br $_{4}$ (yield = $35 \%$ ). M.p. > $300{ }^{\circ} \mathrm{C} .{ }^{1} \mathrm{H}$ NMR $\left[500 \mathrm{MHz}, \mathrm{CDCl}_{3}, \delta\right]: 8.75(\mathrm{~s}, 4 \mathrm{H}), 4.26$ (t, J = 7.05 $\mathrm{Hz}, 4 \mathrm{H}), 4.13(\mathrm{t}, \mathrm{J}=6.25 \mathrm{~Hz}, 4 \mathrm{H}), 2.04(\mathrm{~m}, 4 \mathrm{H}), 1.8(\mathrm{~s}, 6 \mathrm{H}) .{ }^{13} \mathrm{C} \mathrm{NMR}\left[125 \mathrm{MHz}, \mathrm{CDCl}_{3}, \delta\right]: 171.06$, 162.20, 136.21, 131.81, 131.45, 124.05, 122.55, 62.09, 37.99, 27.36, 20.93, IR $\left(\mathrm{KBr}, \mathrm{cm}^{-1}\right): 3041,1675$ and 1249. Elemental analysis: calcd. value for $\mathrm{C}_{34} \mathrm{H}_{22} \mathrm{Br}_{4} \mathrm{~N}_{2} \mathrm{O}_{8}: 45.07 \% \mathrm{C}, 2.45 \% \mathrm{H}$ and $3.09 \% \mathrm{~N}$; found: $45.18 \% \mathrm{C}, 2.60 \% \mathrm{H}$ and $3.05 \% \mathrm{~N}$. HRMS (ESI) $\mathrm{m} / \mathrm{z}$ calculated for $\mathrm{C}_{34} \mathrm{H}_{22} \mathrm{Br}_{4} \mathrm{~N}_{2} \mathrm{O}_{8}[\mathrm{M}]^{+}$: 906.1631, found: 906.1724 .

Table S1. Single crystal analysis of PDI-Br $2-4$

\begin{tabular}{|c|c|c|c|}
\hline & $\mathrm{PDI}_{-\mathrm{Br}_{2}}$ & $\mathrm{PDI}_{-\mathrm{Br}_{3}}$ & $\mathrm{PDI}_{-\mathrm{Br}_{4}}$ \\
\hline formula & $\mathrm{C}_{34} \mathrm{H}_{24} \mathrm{Br}_{2} \mathrm{~N}_{2} \mathrm{O}_{8}$ & $\mathrm{C}_{34} \mathrm{H}_{23} \mathrm{Br}_{3} \mathrm{~N}_{2} \mathrm{O}_{8}$ & $\mathrm{C}_{34} \mathrm{H}_{22} \mathrm{Br}_{4} \mathrm{~N}_{2} \mathrm{O}_{8}$ \\
\hline formula wt & 748.3710 & 827.2610 & 906.1631 \\
\hline colour, shape & Red, Needle & Red, Block & Red, Block \\
\hline crystal system & Triclinic & Triclinic & Triclinic \\
\hline space group, & P-1 & $\mathrm{P}-1$ & $\mathrm{P}-1$ \\
\hline $\mathrm{a}, \AA$ & $4.8146(7)$ & $11.4678(6)$ & $10.800(5)$ \\
\hline $\mathrm{b}, \AA$ & $9.1602(14)$ & $12.2449(6)$ & $11.197(5)$ \\
\hline $\mathrm{c}, \AA$ & $17.672(3)$ & $12.6152(6)$ & $13.581(5)$ \\
\hline$\alpha, \operatorname{deg}$ & $76.925(5)$ & $84.037(2)$ & $81.322(5)$ \\
\hline$\beta$, deg & $84.870(5)$ & $82.349(2)$ & $77.257(5)$ \\
\hline$\gamma$, deg & $75.057(5)$ & $70.582(2)$ & $89.597(5)$ \\
\hline $\mathrm{V}, \AA^{3}$ & 733.11(19) & $1652.44(14)$ & $1582.9(12)$ \\
\hline temp, $\mathrm{K}$ & 296 & 296 & 296 \\
\hline $\mathrm{d}_{\text {calcd }}, \mathrm{g} / \mathrm{cm}^{-3}$ & 1.694 & 1.820 & 1.900 \\
\hline no. of reflections collected & 10852 & 5794 & 5384 \\
\hline no. of unique reflections & 4884 & 4441 & 3956 \\
\hline $2 \theta_{\max }, \mathrm{deg}$ & 50 & 50 & 50 \\
\hline no. of parameters & 218 & 433 & 435 \\
\hline $\mathrm{R} 1$, wR2 $(\mathrm{I}>2 \sigma(\mathrm{I}))$ & $0.0443,0.1260$ & $0.0985,0.3155$ & $0.0305,0.0719$ \\
\hline R1, wR2 (all data) & $0.0596,0.1463$ & $0.1217,0.3374$ & $0.0563,0.1038$ \\
\hline goodness of fit & 1.142 & 1.116 & 1.083 \\
\hline CCDC number & 1402604 & 1402605 & 1402606 \\
\hline
\end{tabular}


Table S2. Electrochemical studies of derivatives PDI-Br ${ }_{0-4}$

\begin{tabular}{ccc}
\hline Derivative & $\mathrm{E}_{\text {red1 }}(\mathrm{V})$ & $\mathrm{E}_{\text {red2 }}(\mathrm{V})$ \\
\hline PDI & -0.547 & -0.728 \\
PDI-Br & -0.517 & -0.707 \\
PDI-Br & -0.502 & -0.706 \\
PDI-Br $_{3}$ & -0.393 & -0.591 \\
PDI-Br $_{4}$ & -0.319 & -0.528 \\
\hline
\end{tabular}

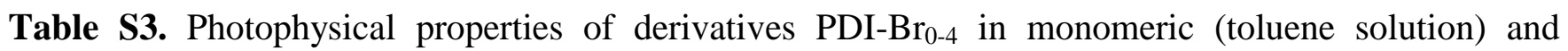
polycrystalline state

\begin{tabular}{|c|c|c|c|c|c|c|c|c|c|}
\hline & \multicolumn{6}{|c|}{ Solution (Toluene) } & \multicolumn{3}{|c|}{ Polycrystalline thin film } \\
\hline & $\lambda_{\mathrm{abs}}, \mathrm{nm}$ & $\begin{array}{c}\varepsilon_{\text {molar }}, \\
\mathrm{Lmol}^{-1} \mathrm{~cm}^{-1}\end{array}$ & $\lambda_{\mathrm{em}}, \mathrm{nm}$ & $\tau_{\mathrm{f}}, \mathrm{ns}$ & $\Phi_{\mathrm{f}}, \%$ & $\Phi_{\mathrm{T}}, \%$ & $\lambda_{\mathrm{abs}}, \mathrm{nm}$ & $\lambda_{\mathrm{em}}, \mathrm{nm}$ & $\Phi_{\mathrm{f}}, \%$ \\
\hline PDI & $\begin{array}{c}459,490 \\
525\end{array}$ & 71000 & $\begin{array}{l}536,577 \\
627\end{array}$ & 4.17 & $\begin{array}{c}97 \\
(99)^{*}\end{array}$ & $<1$ & $\begin{array}{c}390,470,500 \\
555,590\end{array}$ & $\begin{array}{l}\text { 650-800 } \\
\text { (bs) }\end{array}$ & 1 \\
\hline PDI-Br & $\begin{array}{c}459,490, \\
526\end{array}$ & 60088 & $\begin{array}{l}543,582 \\
630\end{array}$ & 4.49 & $\begin{array}{c}97 \\
(97)^{*}\end{array}$ & $<1$ & $\begin{array}{c}400,475,495 \\
545\end{array}$ & 635 & 2 \\
\hline $\mathrm{PDI}-\mathrm{Br}_{2}$ & $\begin{array}{c}460,491, \\
526\end{array}$ & 51587 & $\begin{array}{c}550,588 \\
640\end{array}$ & 4.73 & $\begin{array}{c}94 \\
(96)^{*}\end{array}$ & 1 & $400,500,540$ & 610 & 3 \\
\hline $\mathrm{PDI}-\mathrm{Br}_{3}$ & $\begin{array}{r}425,462, \\
494,530\end{array}$ & 39801 & 561,603 & 4.89 & $\begin{array}{c}85 \\
(83)^{*}\end{array}$ & 5 & $425,500,535$ & 595 & 7 \\
\hline PDI-Br 4 & $\begin{array}{r}440,465, \\
499,532\end{array}$ & 26743 & 570,610 & 4.13 & $\begin{array}{c}64 \\
(64)^{*}\end{array}$ & 18 & $440,505,550$ & 570,620 & 12 \\
\hline
\end{tabular}

*Quantum yield determined in 1,2-dibromoethane confirms the absence of external heavy atom effect imparted by bromine atoms; bs-broad spectra.

Table S4. Oscillator strength for different transitions of PDI and PDI-Br 4 calculated at TD-B3LYP/6$311 \mathrm{G}+(\mathrm{d}, \mathrm{p})$ level of theory.

\begin{tabular}{lll}
\hline Derivative & \multicolumn{2}{c}{ Excitation } \\
\cline { 2 - 3 } & Transition & Oscillator Strength $(f)$ \\
\hline PDI & $\mathrm{S}_{0} \rightarrow \mathrm{S}_{1}$ & 0.7360 \\
& $\mathrm{~S}_{0} \rightarrow \mathrm{S}_{2}$ & 0.0001 \\
& $\mathrm{~S}_{0} \rightarrow \mathrm{S}_{3}$ & 0.0000 \\
& & \\
PDI-Br & $\mathrm{S}_{0} \rightarrow \mathrm{S}_{1}$ & 0.6433 \\
& $\mathrm{~S}_{0} \rightarrow \mathrm{S}_{2}$ & 0.0004 \\
& $\mathrm{~S}_{0} \rightarrow \mathrm{S}_{3}$ & 0.0284 \\
\hline
\end{tabular}




\begin{tabular}{|c|c|c|}
\hline \multirow[t]{3}{*}{ PDI-Br ${ }_{2}$} & $\mathrm{~S}_{0} \rightarrow \mathrm{S}_{1}$ & 0.5625 \\
\hline & $\mathrm{S}_{0} \rightarrow \mathrm{S}_{2}$ & 0.0059 \\
\hline & $\mathrm{S}_{0} \rightarrow \mathrm{S}_{3}$ & 0.0302 \\
\hline \multirow[t]{3}{*}{$\mathrm{PDI}_{-\mathrm{Br}_{3}}$} & $\mathrm{~S}_{0} \rightarrow \mathrm{S}_{1}$ & 0.4873 \\
\hline & $\mathrm{S}_{0} \rightarrow \mathrm{S}_{2}$ & 0.0918 \\
\hline & $\mathrm{S}_{0} \rightarrow \mathrm{S}_{3}$ & 0.0153 \\
\hline \multirow[t]{3}{*}{$\mathrm{PDI}^{-\mathrm{Br}_{4}}$} & $\mathrm{~S}_{0} \rightarrow \mathrm{S}_{1}$ & 0.4311 \\
\hline & $\mathrm{S}_{0} \rightarrow \mathrm{S}_{2}$ & 0.1131 \\
\hline & $\mathrm{S}_{0} \rightarrow \mathrm{S}_{3}$ & 0.0216 \\
\hline
\end{tabular}

Table S5. Low-lying excited singlet $\left(\mathrm{S}_{\mathrm{n}}\right)$ and triplet $\left(\mathrm{T}_{\mathrm{n}}\right)$ electronic states of the derivatives PDI-Br $\mathrm{P}_{0-4}$ in $\mathrm{eV}$, calculated at TD-B3LYP/6-311G+ $(\mathrm{d}, \mathrm{p})$ level of theory.

\begin{tabular}{ccccccccccc}
\hline & $\begin{array}{c}\text { PDI } \\
(\lambda, \mathrm{nm})\end{array}$ & \multicolumn{2}{c}{$\begin{array}{c}\text { PDI-Br } \\
(\lambda, \mathrm{nm})\end{array}$} & \multicolumn{2}{c}{$\begin{array}{c}\text { PDI-Br } \\
(\lambda, \mathrm{nm})\end{array}$} & \multicolumn{2}{c}{$\begin{array}{c}\text { PDI-Br } \\
(\lambda, \mathrm{nm})\end{array}$} & \multicolumn{2}{c}{$\begin{array}{c}\mathrm{PDI}_{3}-\mathrm{Br}_{4} \\
(\lambda, \mathrm{nm})\end{array}$} \\
\cline { 2 - 12 } & $\mathrm{S}_{\mathrm{n}}$ & $\mathrm{T}_{\mathrm{n}}$ & $\mathrm{S}_{\mathrm{n}}$ & $\mathrm{T}_{\mathrm{n}}$ & $\mathrm{S}_{\mathrm{n}}$ & $\mathrm{T}_{\mathrm{n}}$ & $\mathrm{S}_{\mathrm{n}}$ & $\mathrm{T}_{\mathrm{n}}$ & $\mathrm{S}_{\mathrm{n}}$ & $\mathrm{T}_{\mathrm{n}}$ \\
\hline $\mathrm{n}=1$ & 2.3395 & 1.2091 & 2.3262 & 1.2575 & 2.2891 & 1.2787 & 2.2542 & 1.2873 & 2.2193 & 1.2874 \\
& $(530)$ & $(1025)$ & $(533)$ & $(986)$ & $(542)$ & $(970)$ & $(550)$ & $(963)$ & $(559)$ & $(963)$ \\
$\mathrm{n}=2$ & 3.1901 & 2.6318 & 3.1582 & 2.5964 & 3.0645 & 2.5335 & 2.9179 & 2.4334 & 2.7585 & 2.3364 \\
& $(389)$ & $(471)$ & $(393)$ & $(478)$ & $(405)$ & $(489)$ & $(425)$ & $(510)$ & $(450)$ & $(531)$ \\
$\mathrm{n}=3$ & 3.1946 & 2.7325 & 3.1857 & 2.6898 & 3.1844 & 2.621 & 3.0240 & 2.4995 & 2.9519 & 2.3878 \\
& $(388)$ & $(454)$ & $(389)$ & $(461)$ & $(389)$ & $(473)$ & $(410)$ & $(496)$ & $(420)$ & $(524)$ \\
\hline
\end{tabular}

Table S6. Indicates the efficiency of singlet fission mediated generation of triplet excited state in representative chromophoric systems.

\begin{tabular}{|c|c|c|c|}
\hline & Contributed by & $\begin{array}{c}\text { Molecule } \\
\text { (Reference) }\end{array}$ & $\Phi_{\mathrm{T}}(\%)$ \\
\hline 1 & Our study & Core-twisted tetrabromo perylenediimide & 79 \\
\hline 2 & Campos and coworkers & $\begin{array}{c}\text { Bipentacene } \\
\text { (J. Am. Chem. Soc., 2015, 137, 8965) }\end{array}$ & 200 \\
\hline 3 & Musser and coworkers & $\begin{array}{l}\text { Carotenoid } \\
\text { (J. Am. Chem. Soc., 2015, 137, 5130) }\end{array}$ & - \\
\hline 4 & Sfeir and coworkers & $\begin{array}{l}\text { Benzodithiophene polymer } \\
\text { (Nat. Mater., 2015, 14, 426) }\end{array}$ & 170 \\
\hline 5 & Yaffe and coworkers & $\begin{array}{c}\text { Hexacene } \\
\text { (J. Am. Chem. Soc., 2014, 136, 10654) }\end{array}$ & 200 \\
\hline
\end{tabular}




\begin{tabular}{|c|c|c|c|}
\hline 6 & Venkatesan and coworkers & $\begin{array}{c}\text { b-hydroxy-vinylimine boron } \\
\text { (Angew. Chem. Int. Ed. 2014, 53, 6378) }\end{array}$ & 118 \\
\hline 7 & Wasielewski and co-workers & $\begin{array}{c}\text { Tetraphenyl Perylenediimide } \\
\text { (J. Am. Chem. Soc., 2013, 135, 14701) }\end{array}$ & 140 \\
\hline 8 & Friend and co-workers & $\begin{array}{l}\text { TIPS-Pentacene } \\
\text { (Nat. Chem., 2013, 5, 1019) }\end{array}$ & 200 \\
\hline 9 & Bradforth and co-workers & $\begin{array}{l}\text { Disordered Acene } \\
\text { (J. Am. Chem. Soc., 2012, 134, 6388) }\end{array}$ & 61 \\
\hline 10 & Michl and co-workers & $\begin{array}{c}\text { 1,3-Diphenylisobenzofuran } \\
\text { (J. Am. Chem. Soc., 2010, 132, 16302) }\end{array}$ & 200 \\
\hline 11 & Tauber and co-workers & $\begin{array}{c}\text { Zeaxanthin } \\
\text { (J. Am. Chem. Soc., 2010, 132, 13988) }\end{array}$ & $90-200$ \\
\hline 12 & Bardeen and co-workers & $\begin{array}{c}\text { Bis-tetracene } \\
\text { (J. Am. Chem. Soc., 2007, 129, 14240) }\end{array}$ & $1-2$ \\
\hline
\end{tabular}

Table S7. Low-lying excited singlet $\left(\mathrm{S}_{\mathrm{n}}\right)$ and triplet $\left(\mathrm{T}_{\mathrm{n}}\right)$ electronic states of $\mathrm{PDI}^{-\mathrm{Br}_{4}}$ dimer and monomer in $\mathrm{eV}$, calculated at TD-B3LYP/ LANL2dz level of theory (corresponding oscillator strength is given in the parenthesis).

\begin{tabular}{ccccc}
\hline \multirow{2}{*}{ No. } & \multicolumn{2}{c}{ Monomer } & \multicolumn{2}{c}{ Slip-stacked dimer } \\
\cline { 2 - 5 } & $\mathrm{S}_{\mathrm{n}}(f)$ & $\mathrm{T}_{\mathrm{n}}$ & $\mathrm{S}_{\mathrm{n}}(f)$ & $\mathrm{T}_{\mathrm{n}}$ \\
\hline $\mathrm{n}=1$ & $2.2983(0.4846)$ & 1.3077 & $2.2537(0.0097)$ & 1.4538 \\
$\mathrm{n}=2$ & $2.8080(0.1213)$ & 2.3875 & $2.2542(0.0001)$ & 1.4540 \\
$\mathrm{n}=3$ & $3.0281(0.0241)$ & 2.4289 & $2.3804(0.0000)$ & 2.2471 \\
$\mathrm{n}=4$ & $3.1220(0.0000)$ & 2.5540 & $2.4124(0.9034)$ & 2.2486 \\
$\mathrm{n}=5$ & $3.1241(0.0000)$ & 2.8255 & $2.8186(0.0000)$ & 2.4402 \\
$\mathrm{n}=6$ & $3.2006(0.0000)$ & 2.9208 & $2.8341(0.1413)$ & 2.4412 \\
$\mathrm{n}=7$ & $3.2151(0.0021)$ & 2.9237 & $2.8929(0.0000)$ & 2.5364 \\
$\mathrm{n}=8$ & $3.2198(0.0049)$ & 3.0547 & $2.9030(0.0530)$ & 2.5382 \\
$\mathrm{n}=9$ & $3.3364(0.0005)$ & 3.0658 & $2.9640(0.0000)$ & 2.6787 \\
$\mathrm{n}=10$ & $3.3907(0.0029)$ & 3.1343 & $2.9677(0.0564)$ & 2.6811 \\
\hline
\end{tabular}




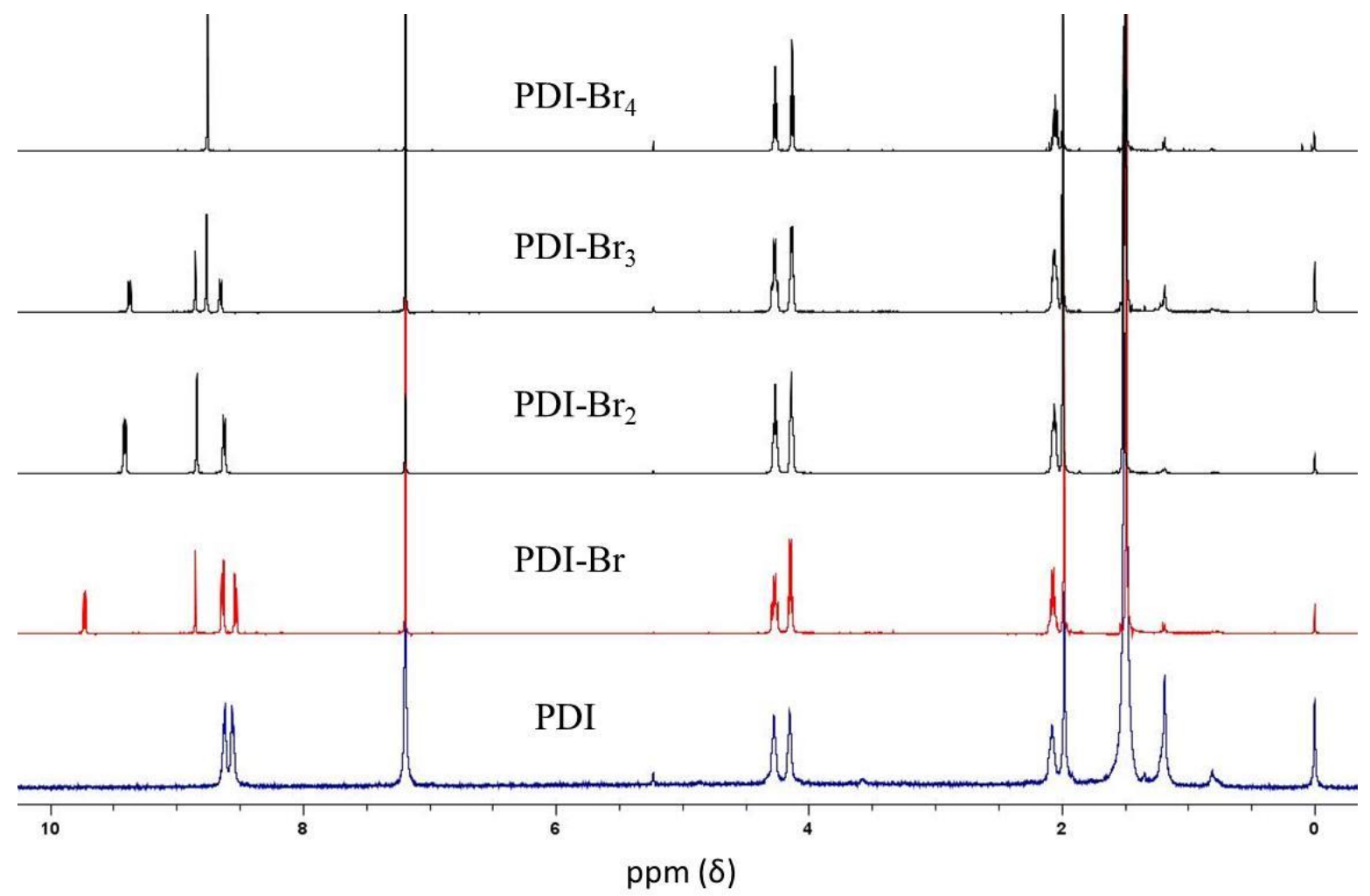

Figure S1. ${ }^{1} \mathrm{H}-\mathrm{NMR}$ spectra of derivatives $\mathrm{PDI}-\mathrm{Br}_{0-4}$ in $\mathrm{CDCl}_{3}$.
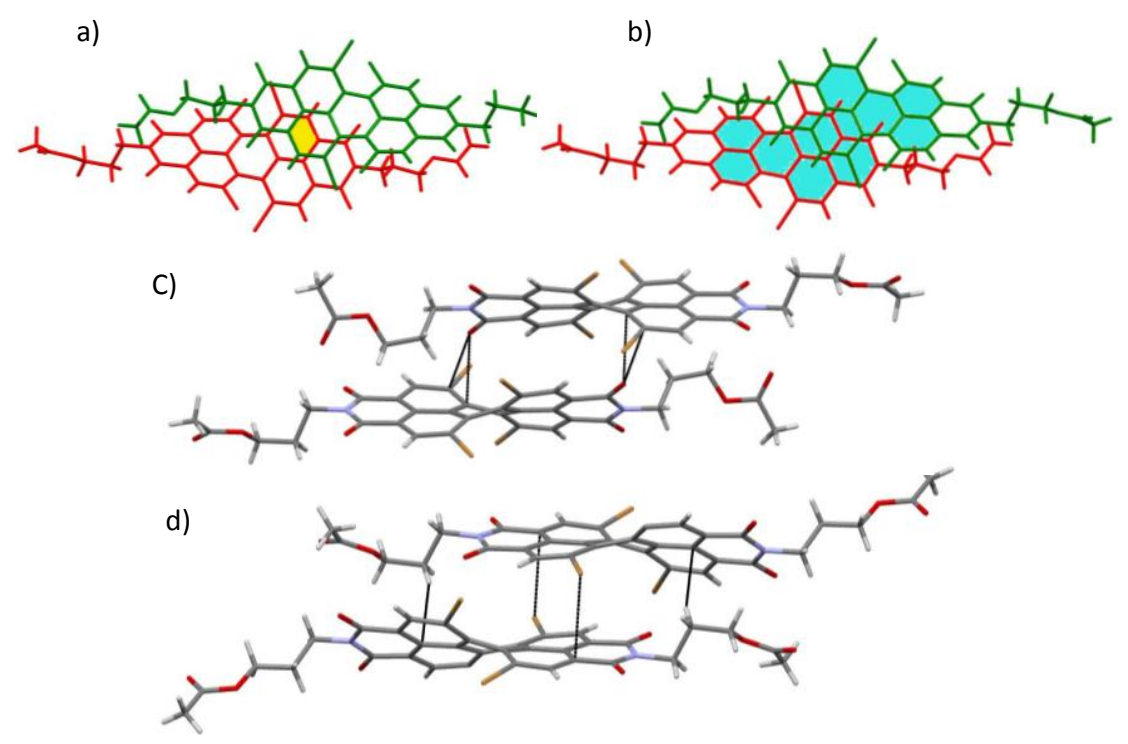

Figure S2. a) Overlapped aromatic area and b) total aromatic surface area between the vicinal perylenediimide moieties of PDI- $\mathrm{Br}_{4}$ obtained from the crystal packing; c) and d) short contact between the vicinal PDI units in PDI-Br 3 and PDI-Br 4 , respectively. 


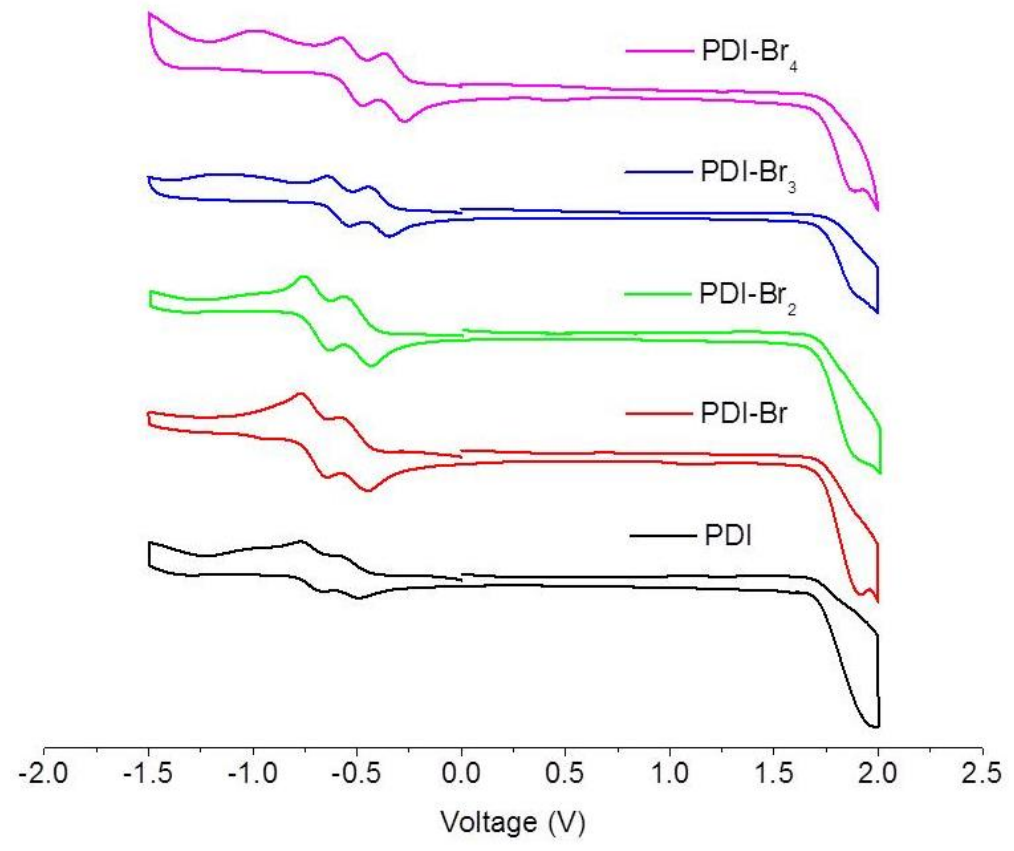

Figure S3. Cyclic voltammogram of $\mathrm{PDI}_{-} \mathrm{Br}_{0-4}$ in dichloromethane with respect to $\mathrm{Ag} / \mathrm{AgCl}$ reference electrode. 


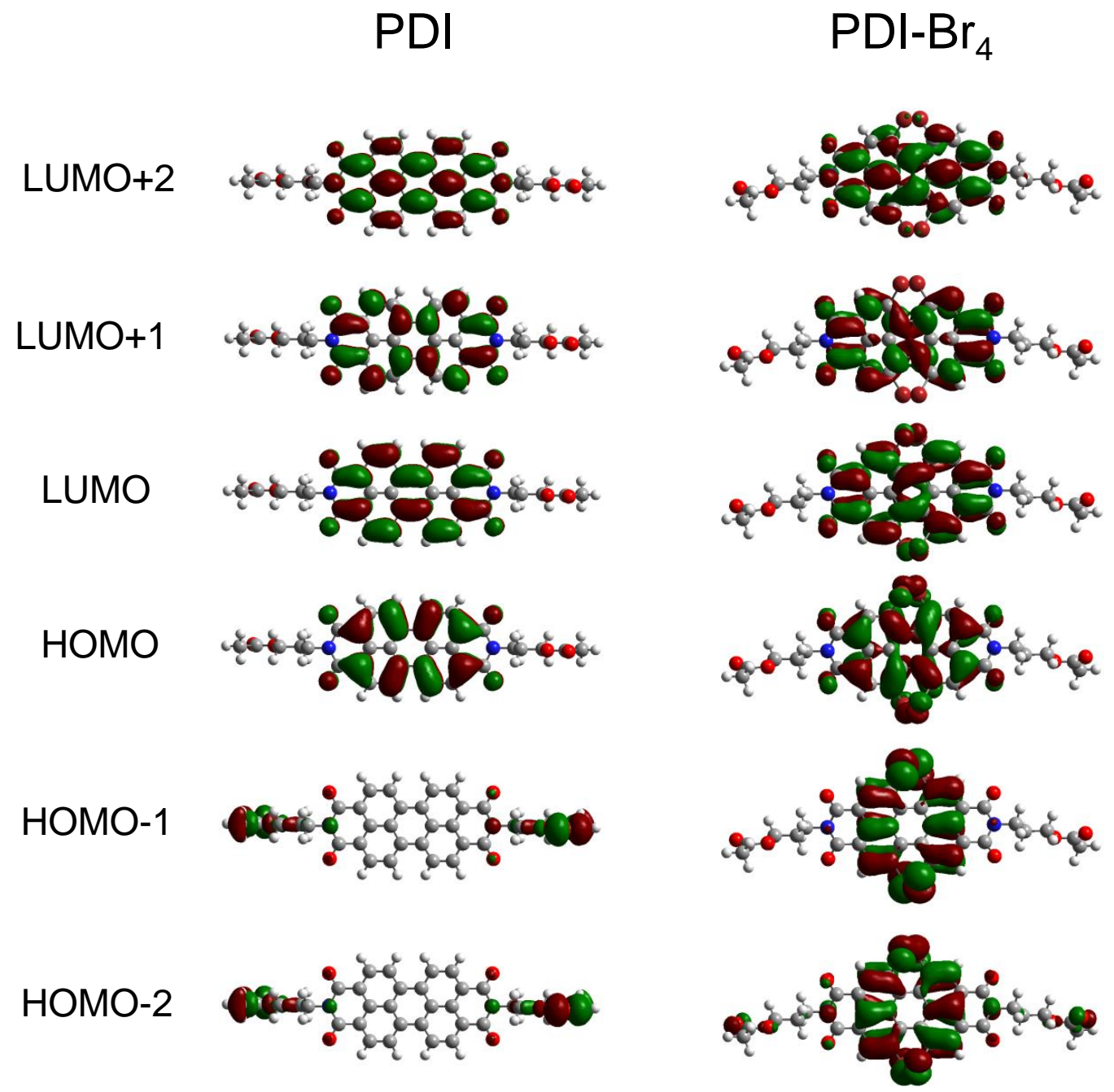

Figure S4. Representative frontier molecular orbital diagrams of the model derivative PDI and the PDI$\mathrm{Br}_{4}$ calculated at TD-B3LYP/6-311G+ $(\mathrm{d}, \mathrm{p})$ level of theory. 


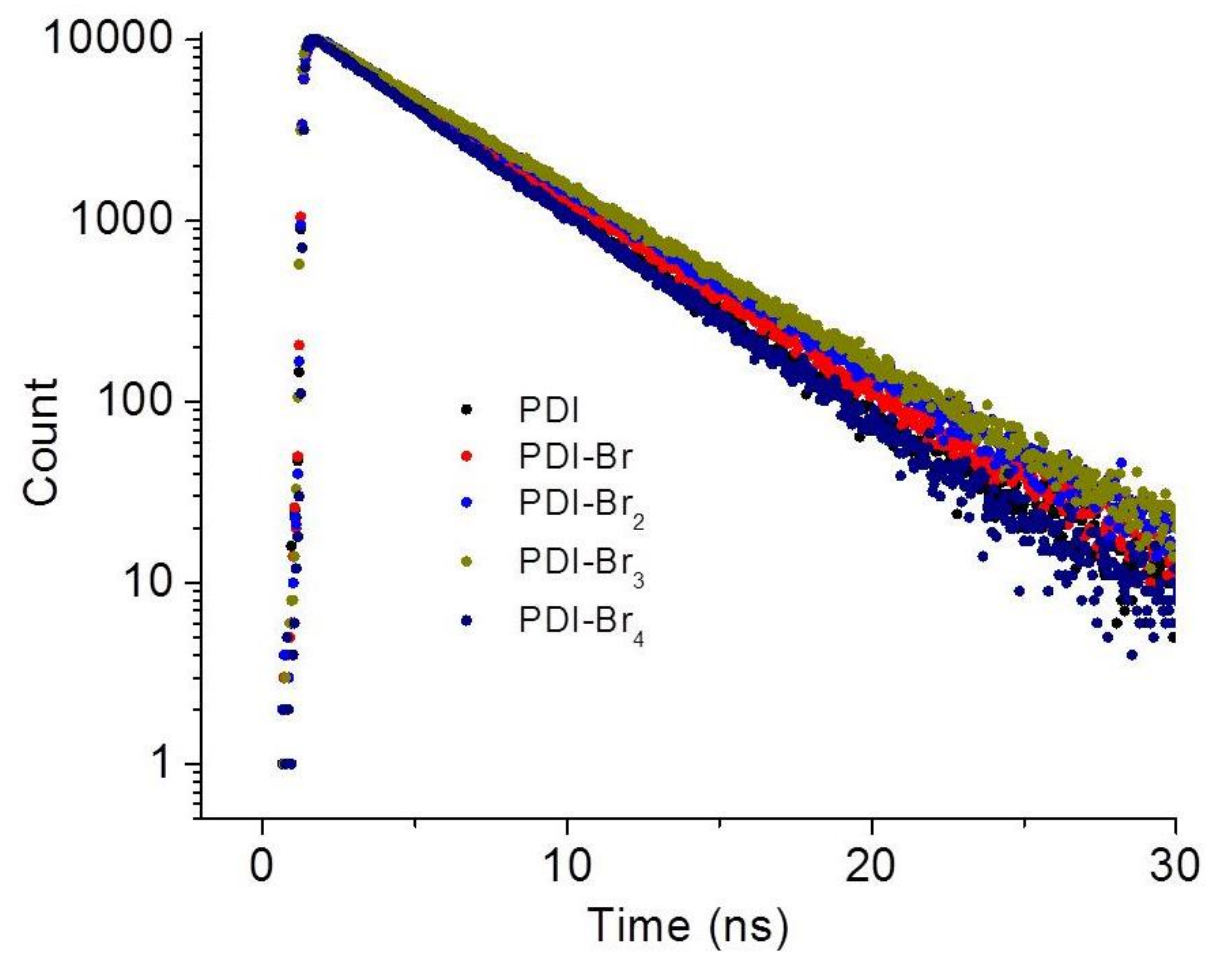

Figure S5. Picosecond time-resolved fluorescence measurements of $\mathrm{PDI}-\mathrm{Br}_{0-4}$ in toluene (excitation wavelength: $439 \mathrm{~nm}$ ) monitored at the respective emission maxima. 

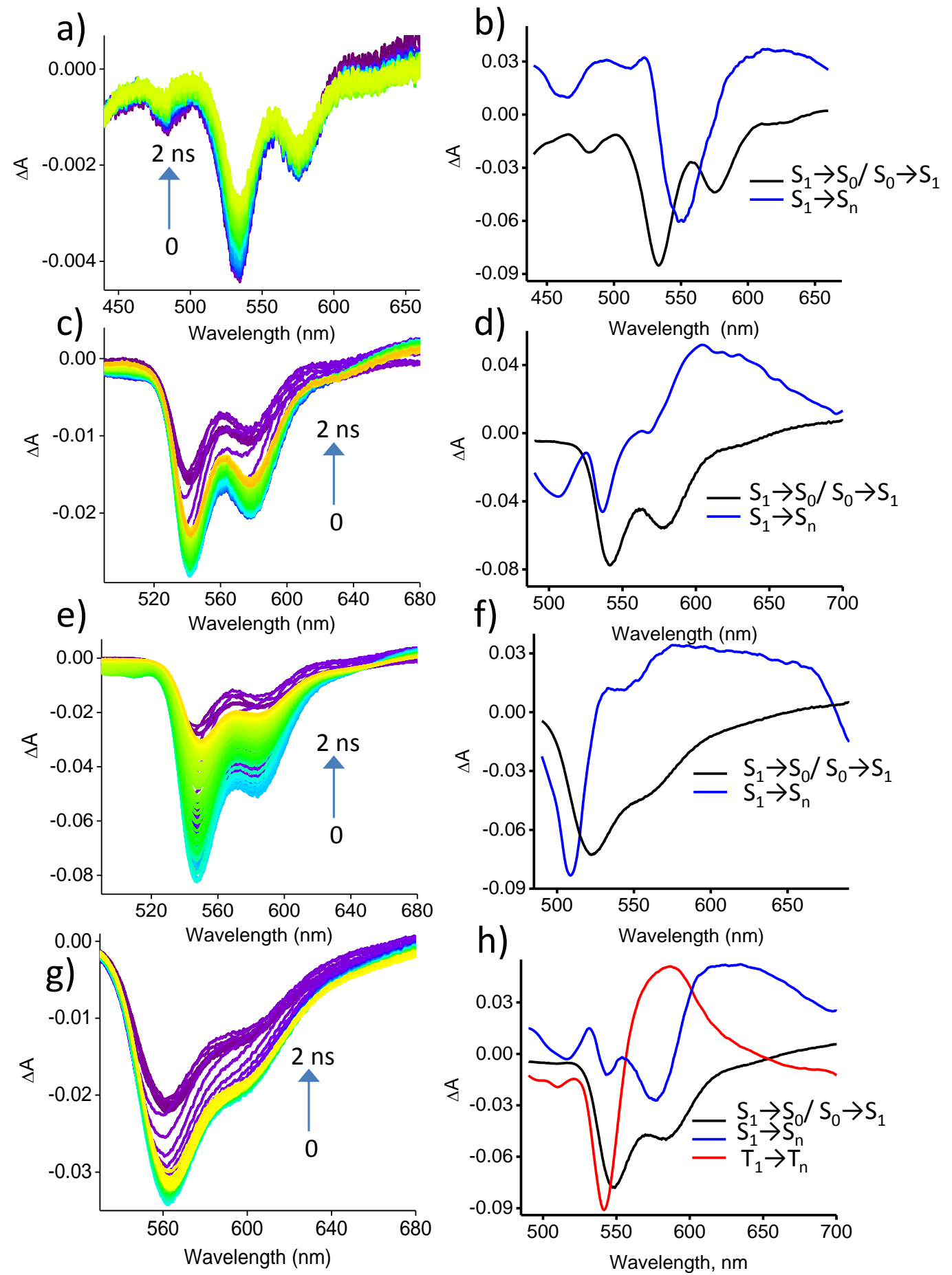

Figure S6. Femtosecond transient absorption spectrum of a) $\mathrm{PDI}$; c) $\mathrm{PDI}-\mathrm{Br}$ e) $\mathrm{PDI}-\mathrm{Br}_{2} \mathrm{~g}$ ) $\mathrm{PDI}^{-\mathrm{Br}_{3}}$; in toluene; excited at $400 \mathrm{~nm} \mathrm{b),} \mathrm{d),} \mathrm{f)} \mathrm{and} \mathrm{h)} \mathrm{are} \mathrm{corresponding} \mathrm{SVD.}$ 

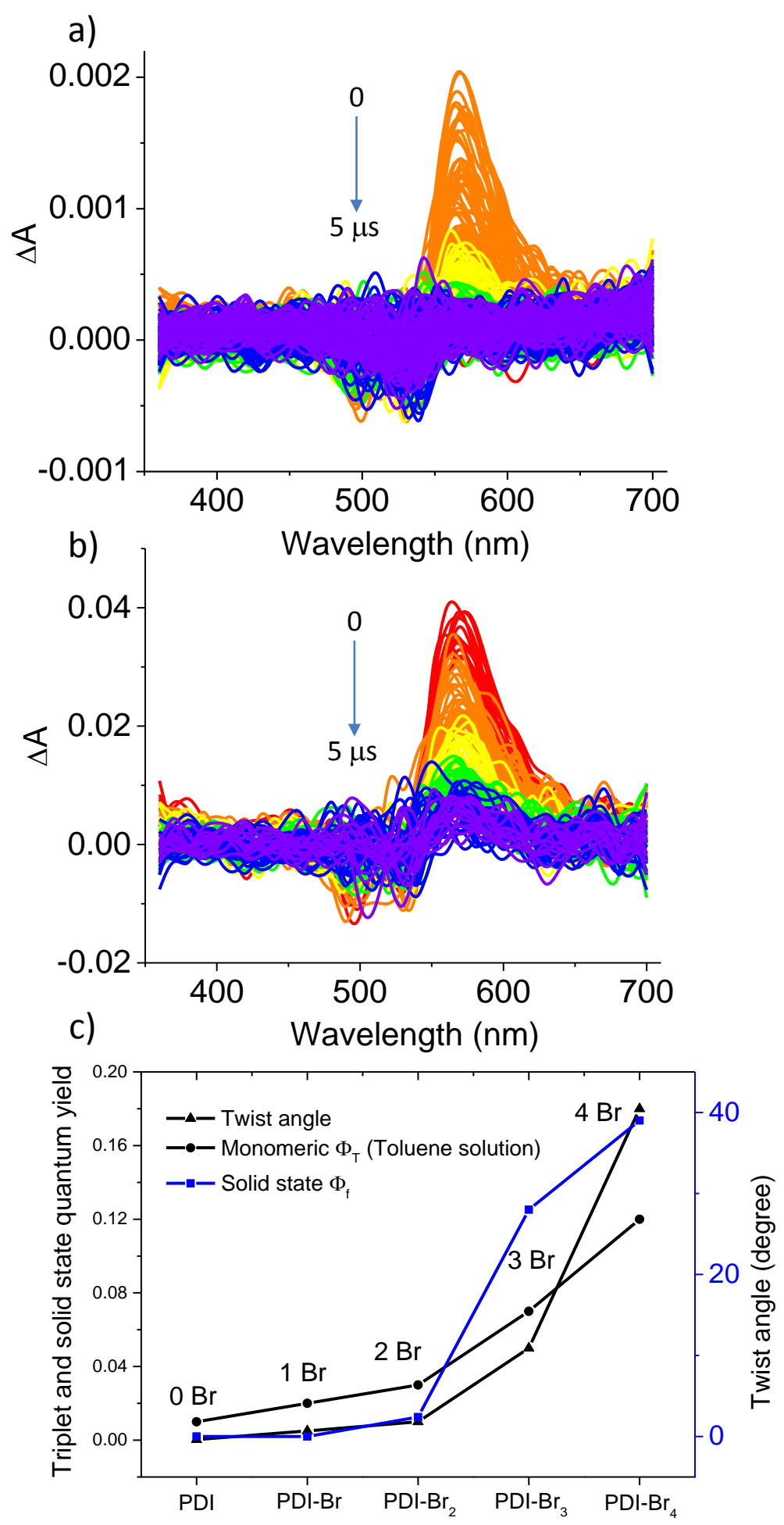

Figure S7. Nanosecond transient absorption spectrum of a) $\mathrm{PDI}-\mathrm{Br}_{2}$ b) $\mathrm{PDI}_{-\mathrm{Br}_{3}}$ in toluene; excited at $532 \mathrm{~nm}$; c) near-quadratic increase in the core-twist angle, triplet quantum yield in toluene solution and solid state fluorescence quantum yield of $\mathrm{PDI}_{-\mathrm{Br}_{0-4}}$ with the linear increase in number of bromine atoms. 


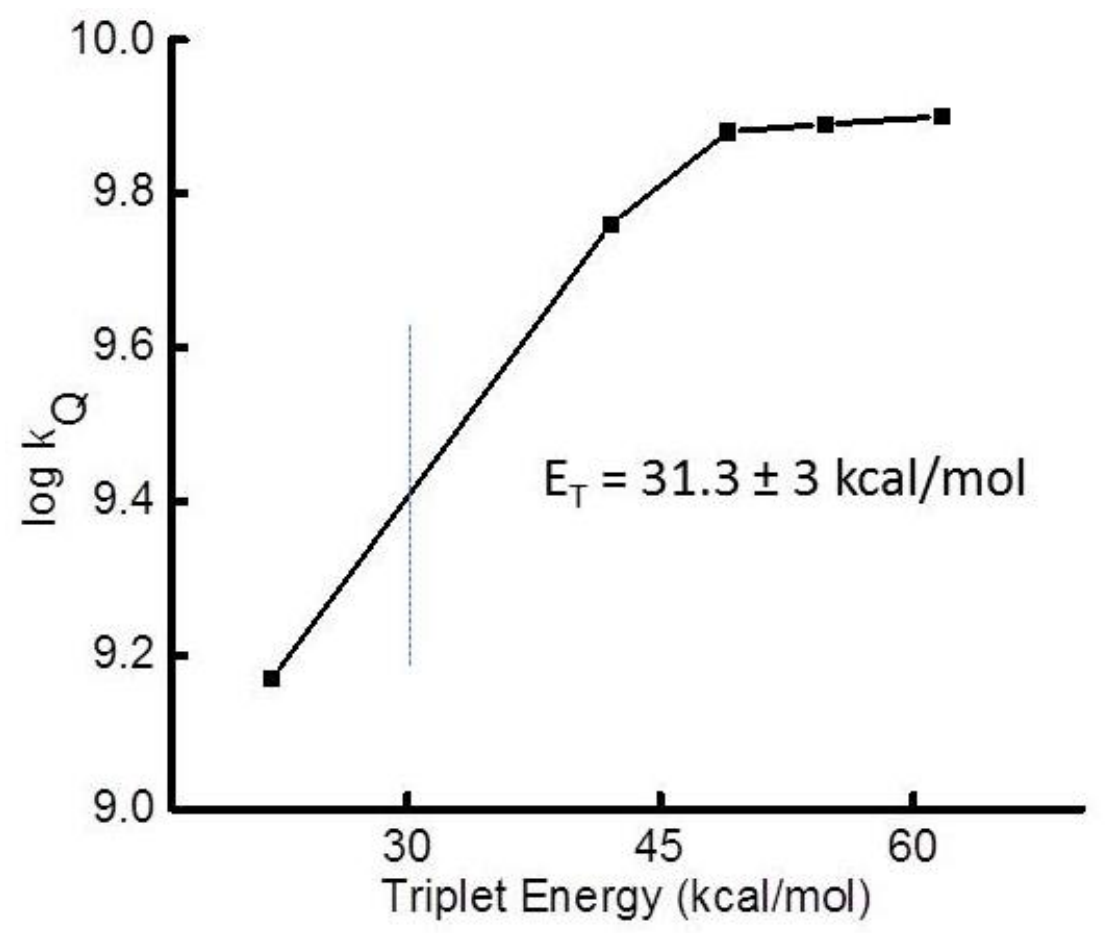

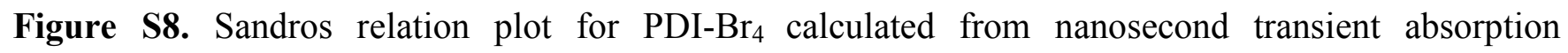
measurements.
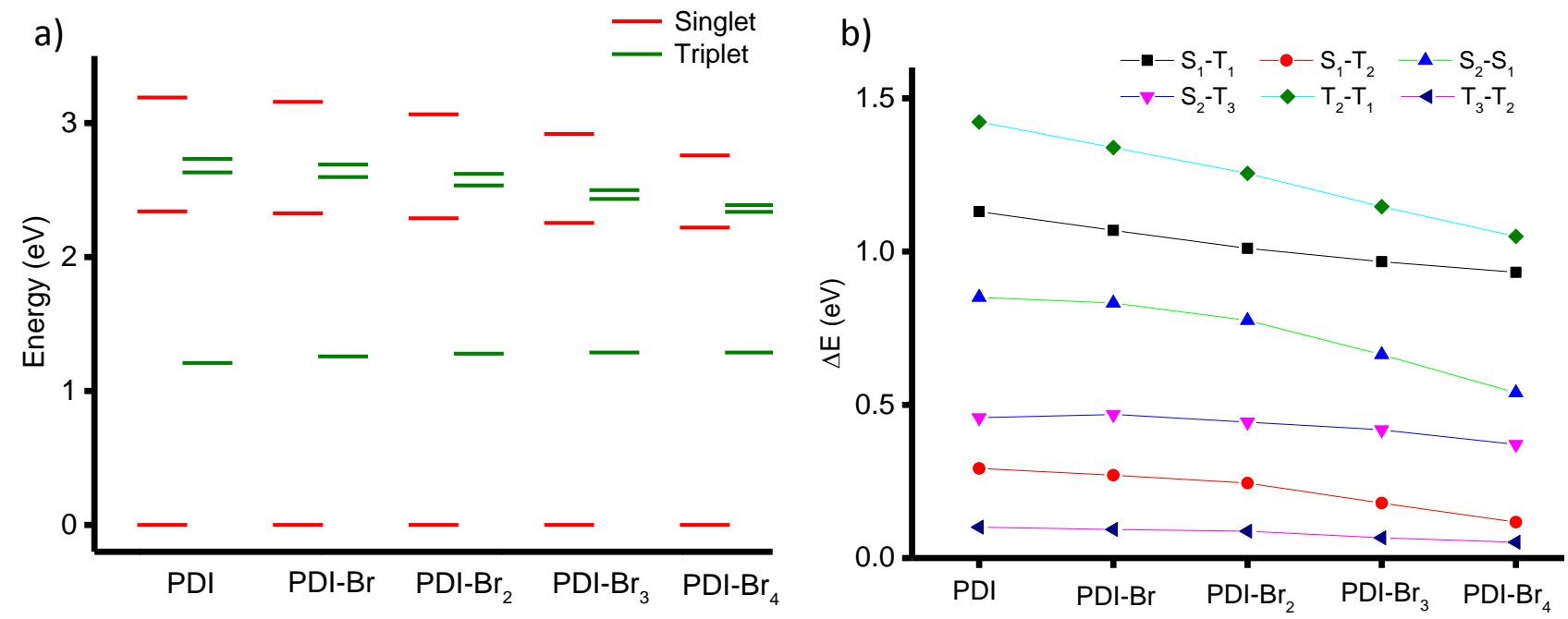

Figure S9. a) Energy level diagram for PDI and PDI-Br Pen $_{-4}$ derivatives calculated at TD-B3LYP/6$311 \mathrm{G}+(\mathrm{d}, \mathrm{p})$ level of theory, b) energy difference between various excited states. 


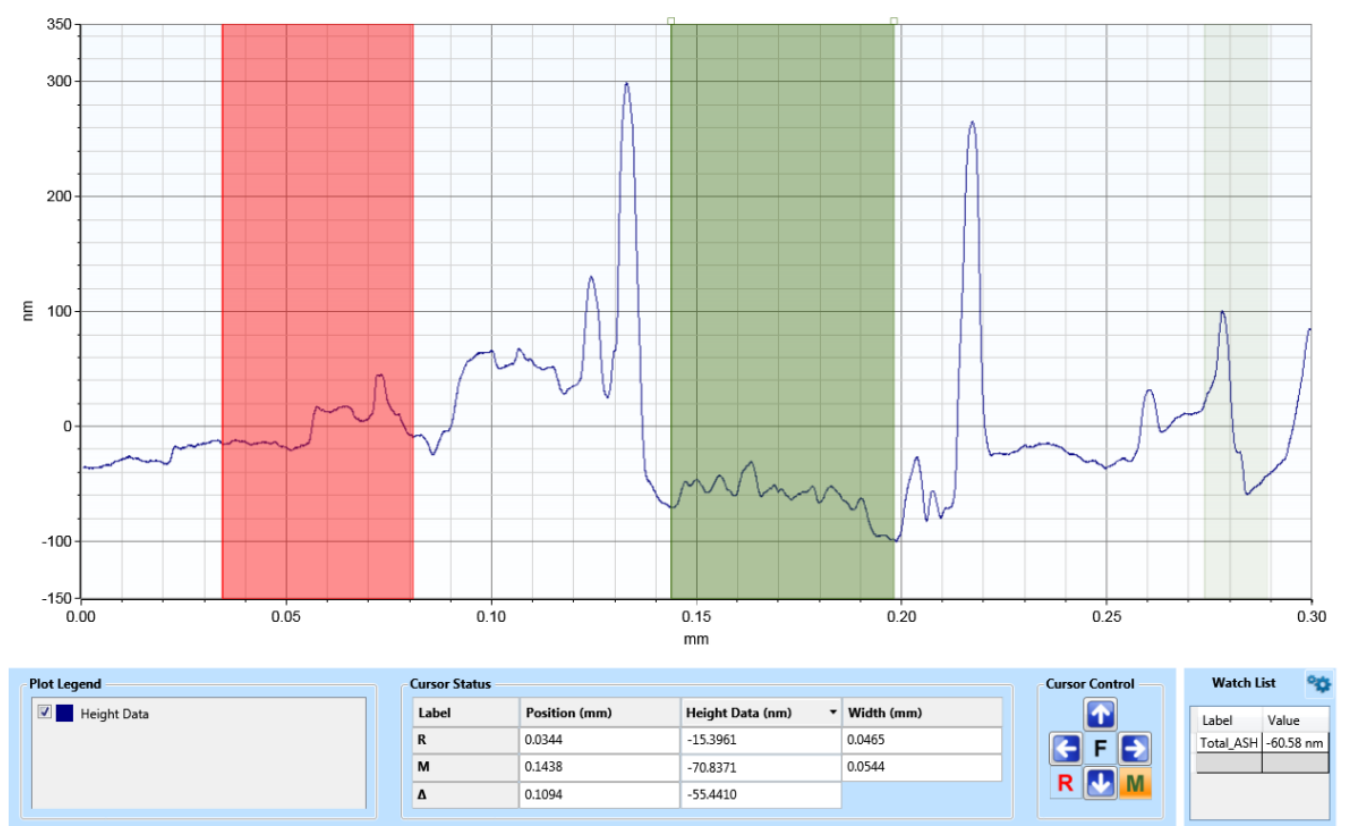

Figure S10. Profilometry trace for PDI-Br 4 thin film. Calculated thickness is $60 \pm 5 \mathrm{~nm}$.

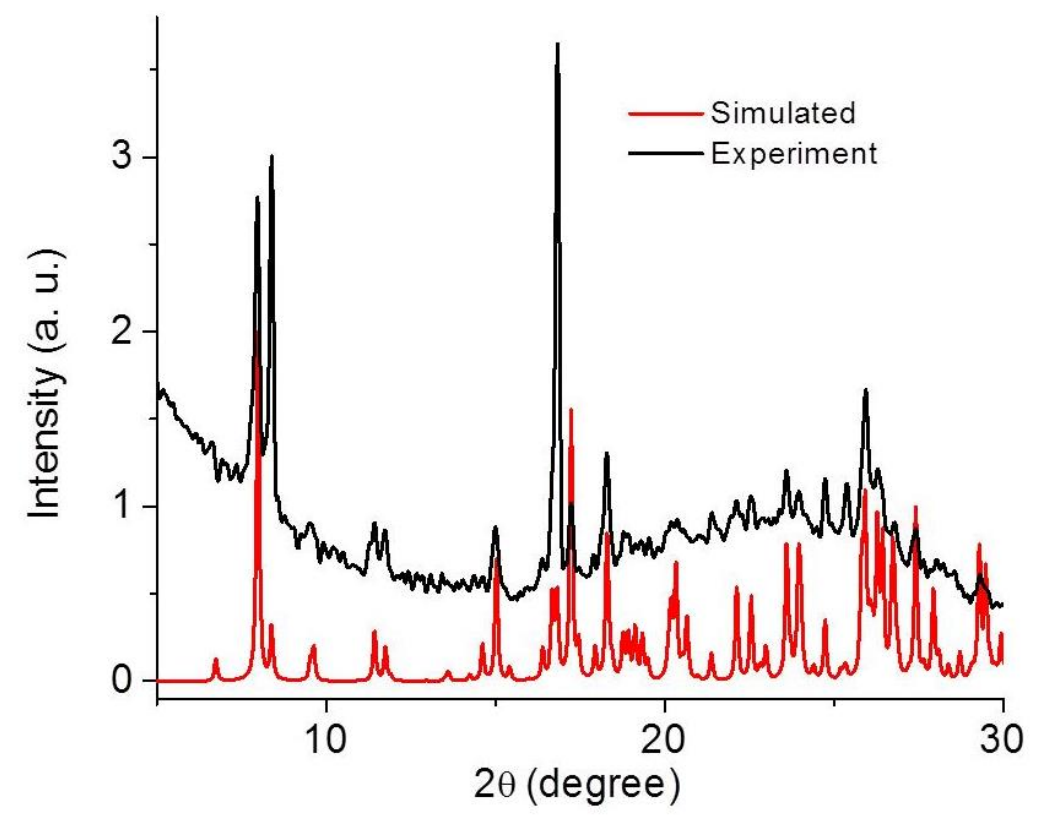

Figure S11. PXRD diffractogram of PDI-Br 4 polycrystalline thin film (black trace) overlaid with the diffractogram simulated from the single crystal structure of $\mathrm{PDI}_{-\mathrm{Br}_{4}}$ (red trace). 


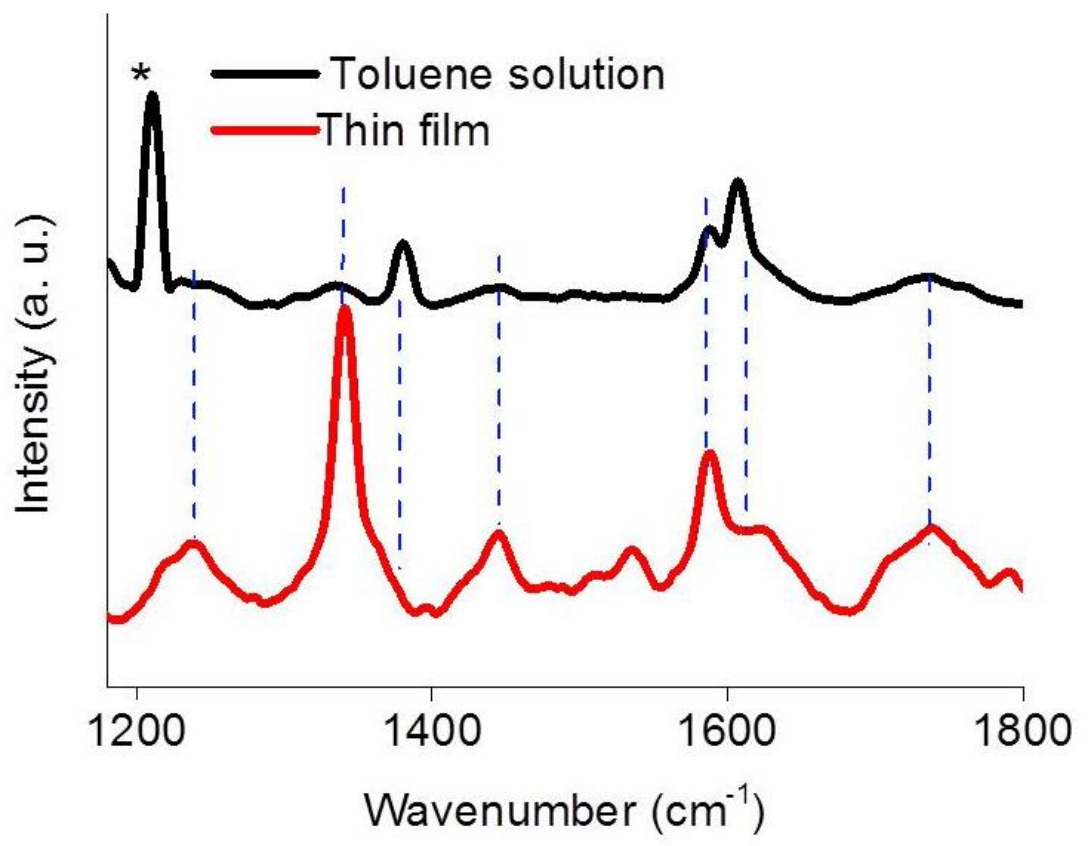

Figure S12. Ground state raman spectra of PDI- $\mathrm{Br}_{4}$ in toluene solution (black trace) and polycrystalline thinfilm state (red trace) on excitation with $480 \mathrm{~nm}$ laser (* denotes toluene absorption band).

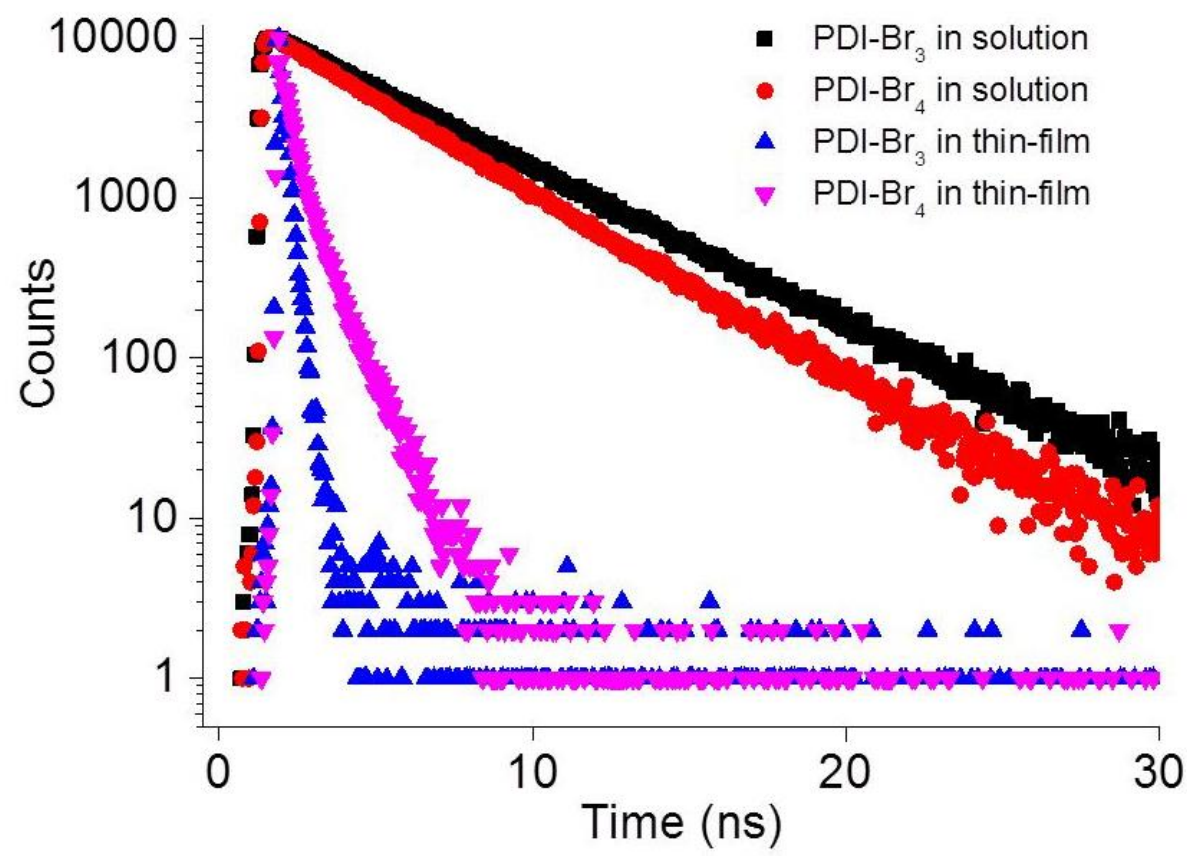

Figure S13. Picosecond time-resolved fluorescence measurements of $\mathrm{PDI}_{-} \mathrm{Br}_{3-4}$ in toluene and in thinfilm state on exciting at $439 \mathrm{~nm}$ and monitored at the respective emission maxima. 

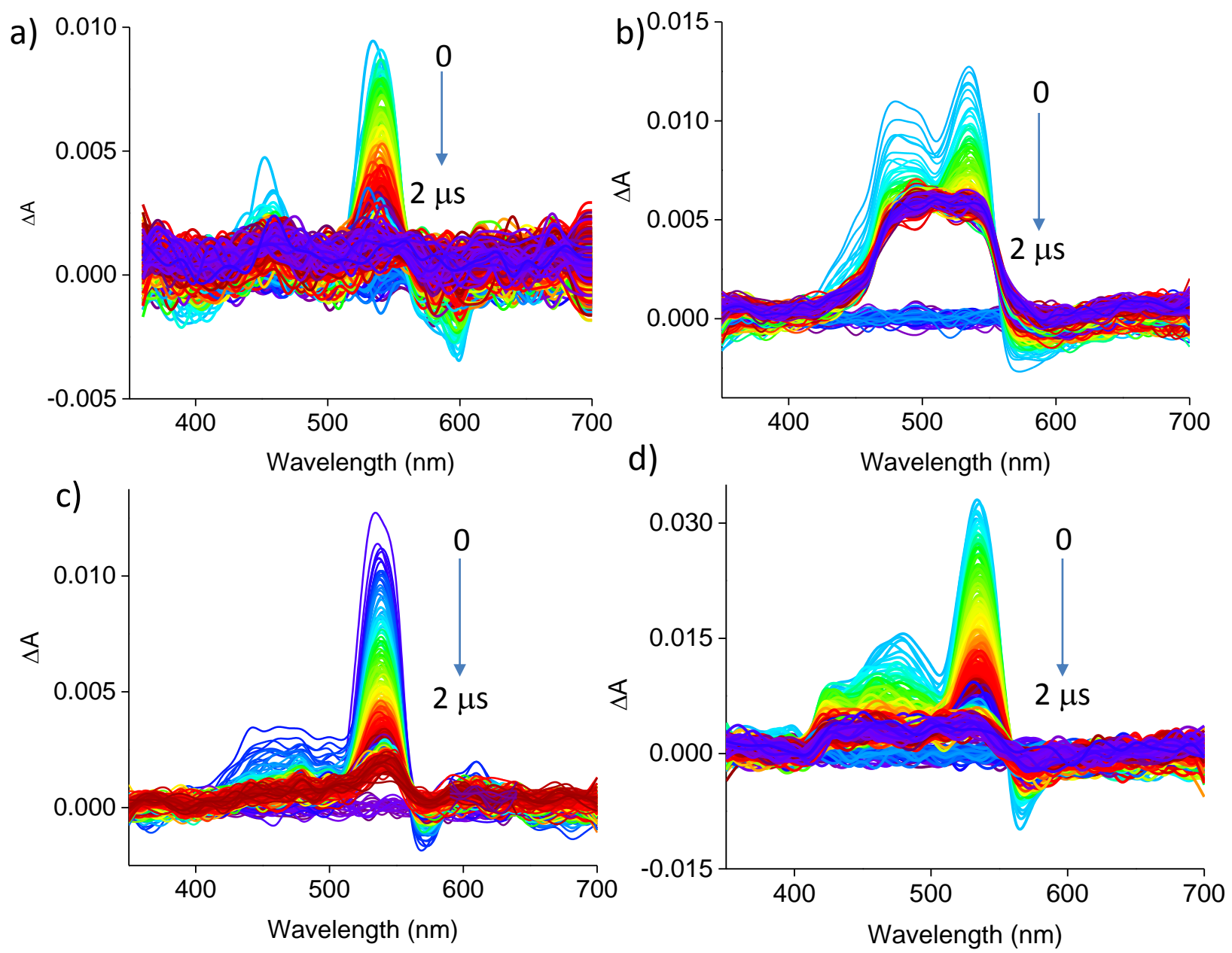

Figure S14. nTA spectra of a) PDI, b) PDI-Br, c) PDI- $-\mathrm{Br}_{2}$ and d) $\mathrm{PDI}-\mathrm{Br}_{3}$ in thin film state.

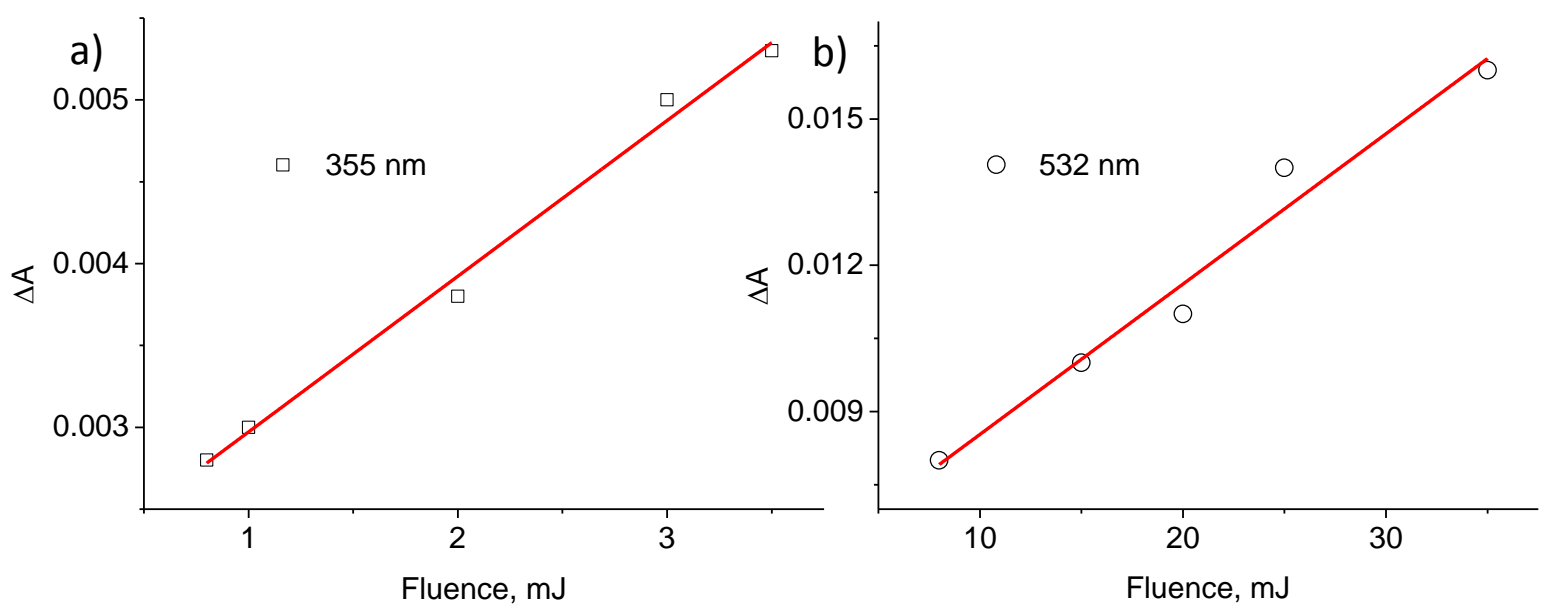

Figure S15. Excitation density dependence of the ground state depletion of polycrystalline PDI-Br 4 on excitation at a) $355 \mathrm{~nm}$ (probed at $590 \mathrm{~nm}$ ) and b) $532 \mathrm{~nm}$ (probed at $590 \mathrm{~nm}$ ). 

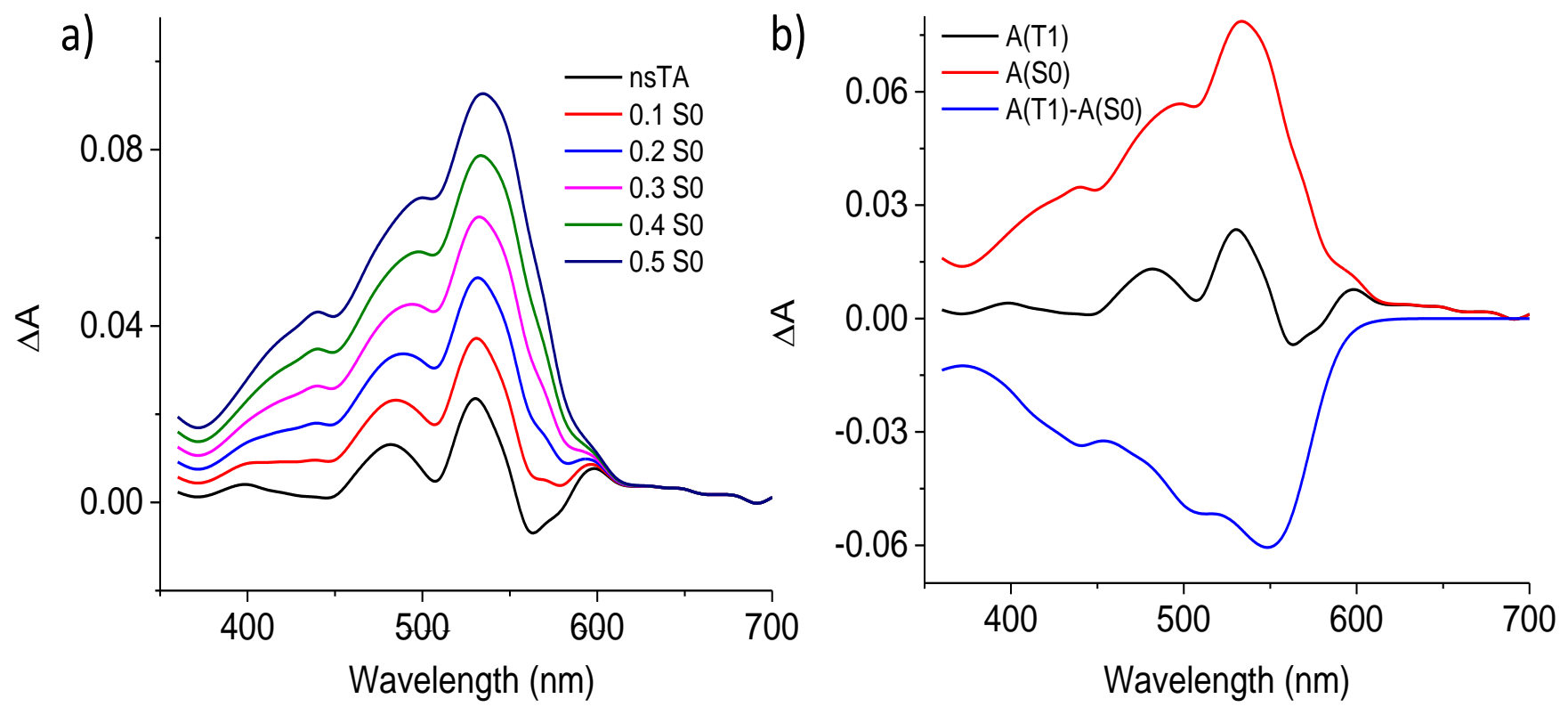

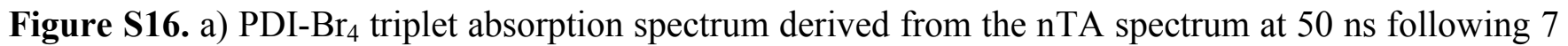
$\mathrm{ns}, 532 \mathrm{~nm}$ laser pulse and the ground state (GS) absorption spectrum in polycrystalline thin film. $\left.{ }^{1} \mathrm{~b}\right)$ the absorbance required to completely account for the ground state bleach is roughly 0.0782 that predicted by the calculation. The observed bleach is 0.0616 . So the calculated triplet yield $=0.0616 / 0.0782=$ $0.788(79 \%)$.
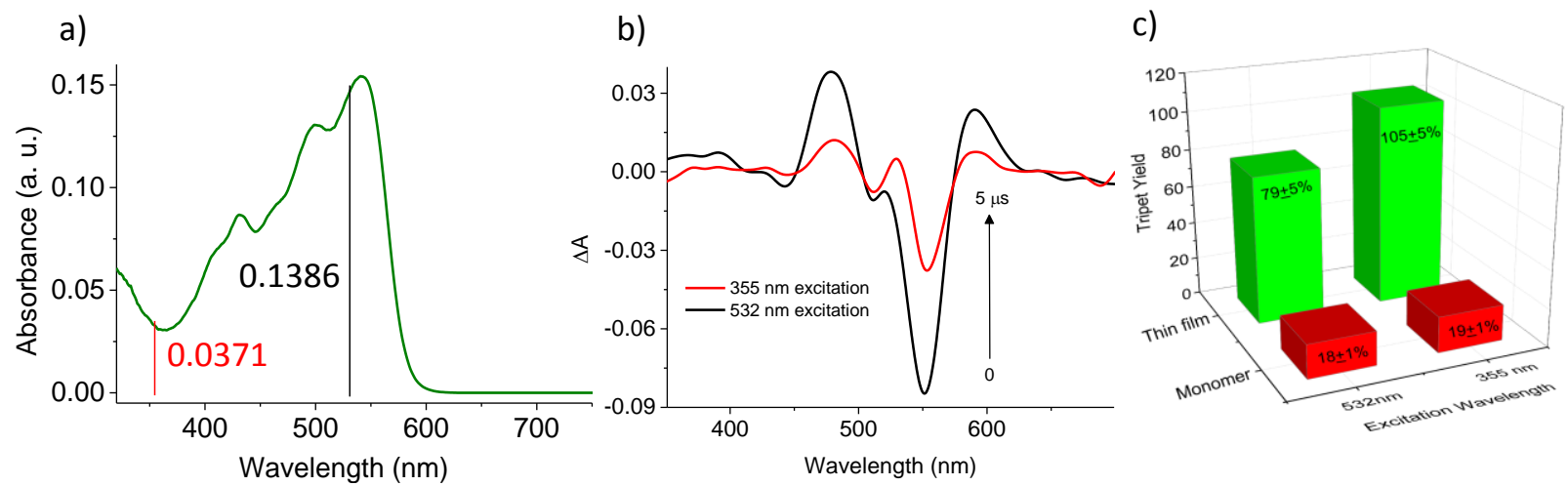

Figure S17. Show a) ground state absorption spectra; b) nTA spectra (excitation wavelength 355 and $532 \mathrm{~nm}$ ) of polycrystalline thin film of $\mathrm{PDI}_{-\mathrm{Br}_{4}}$; c) relative quantum yield of triplet generation in PDI$\mathrm{Br}_{4}$ polycrystalline thin film on exciting at 355 and $532 \mathrm{~nm}$. 

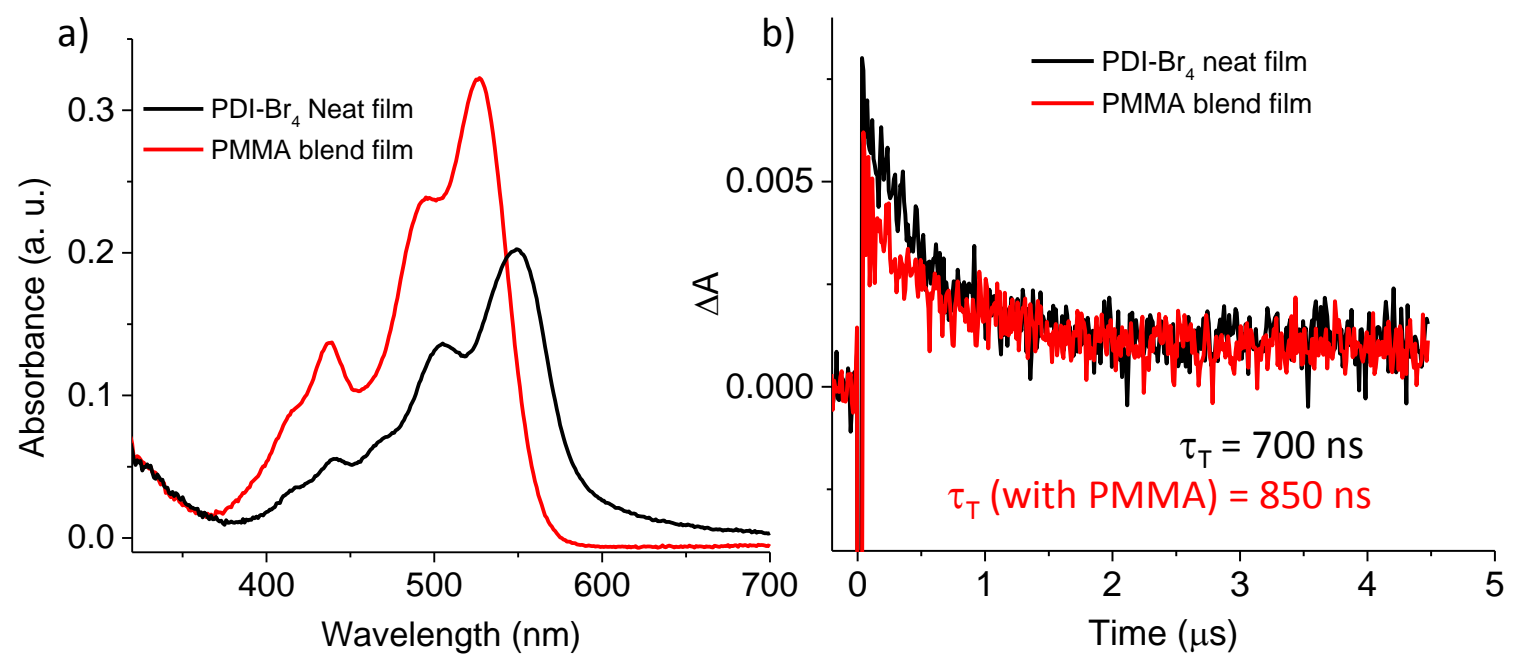

Figure S18. Show a) ground state absorption spectra; b) relative optical density and decay profile of nTA at $590 \mathrm{~nm}$ (excitation wavelength $355 \mathrm{~nm}$ ) of PDI-Br 4 neat and PMMA blend thin film.

$\underline{\text { PDI ground state optimized coordinates calculated at B3LYP/6-31+G(d,p) level of theory }}$

$\begin{array}{lrrr}\mathrm{C} & 1.46027600 & 2.43193200 & 0.24882000 \\ \mathrm{C} & 0.72519000 & 1.25011800 & 0.12450200 \\ \mathrm{C} & -0.72521500 & 1.25010600 & -0.12454100 \\ \mathrm{C} & -1.46031300 & 2.43190900 & -0.24889000 \\ \mathrm{C} & 1.41256900 & 0.00005400 & 0.24379300 \\ \mathrm{C} & -1.41257600 & 0.00003100 & -0.24381100 \\ \mathrm{C} & -0.72518000 & -1.25003400 & -0.12463100 \\ \mathrm{C} & 0.72518800 & -1.25002300 & 0.12463600 \\ \mathrm{C} & -1.46021400 & -2.43184600 & -0.24927100 \\ \mathrm{C} & -2.83960100 & -2.42394200 & -0.48736000 \\ \mathrm{C} & -2.82239700 & 0.00002200 & -0.48782400 \\ \mathrm{C} & -3.52391600 & 1.22539400 & -0.60833500 \\ \mathrm{C} & -2.83970800 & 2.42398600 & -0.48693200 \\ \mathrm{C} & 2.83967400 & 2.42403000 & 0.48684900 \\ \mathrm{C} & 3.52389800 & 1.22544900 & 0.60827100 \\ \mathrm{C} & 2.82239100 & 0.00006600 & 0.48779600 \\ \mathrm{C} & 2.83962500 & -2.42389800 & 0.48738200 \\ \mathrm{C} & 1.46023800 & -2.43182400 & 0.24929100 \\ \mathrm{C} & -4.98417100 & 1.24292600 & -0.86142300 \\ \mathrm{C} & 3.52387400 & -1.22530500 & 0.60853400 \\ \mathrm{C} & 4.98413000 & -1.24283100 & 0.86161300 \\ \mathrm{C} & 4.98415900 & 1.24300600 & 0.86132300 \\ \mathrm{O} & 5.62222300 & 2.28546200 & 0.96286400 \\ \mathrm{O} & 5.62217400 & -2.28528100 & 0.96335200 \\ \mathrm{O} & -5.62224500 & 2.28537400 & -0.96298900 \\ \mathrm{C} & -3.52386600 & -1.22536000 & -0.60853400 \\ \mathrm{C} & -4.98412400 & -1.24291100 & -0.86160000 \\ \mathrm{O} & -5.62215800 & -2.28537000 & -0.96330500 \\ \mathrm{~N} & -5.62308300 & 0.00000500 & -0.98632100 \\ \mathrm{~N} & 5.62307700 & 0.00009600 & 0.98629400 \\ \mathrm{H} & -0.96930300 & 3.39302700 & -0.16103000 \\ \mathrm{H} & 0.96925500 & 3.39304200 & 0.16094200 \\ \mathrm{H} & 0.96919400 & -3.39294400 & 0.16165600\end{array}$




$\begin{array}{lccc}\mathrm{H} & -0.96915700 & -3.39295900 & -0.16162700 \\ \mathrm{H} & 3.39153900 & -3.35301800 & 0.58015800 \\ \mathrm{H} & -3.39150400 & -3.35307100 & -0.58011600 \\ \mathrm{H} & 3.39161500 & 3.35315900 & 0.57937100 \\ \mathrm{H} & -3.39165900 & 3.35310600 & -0.57948800 \\ \mathrm{C} & 7.07312600 & 0.00011200 & 1.25039300 \\ \mathrm{H} & 7.28504400 & 0.89249400 & 1.84155100 \\ \mathrm{H} & 7.28502200 & -0.89210000 & 1.84180900 \\ \mathrm{C} & 7.89374500 & -0.00006600 & -0.04599000 \\ \mathrm{H} & 7.64880100 & -0.88622800 & -0.63997600 \\ \mathrm{C} & -7.07313600 & -0.00000300 & -1.25040000 \\ \mathrm{H} & -7.28503600 & -0.89227400 & -1.84172800 \\ \mathrm{H} & -7.28506200 & 0.89232100 & -1.84164100 \\ \mathrm{C} & -7.89373600 & -0.00006900 & 0.04599500 \\ \mathrm{H} & -7.64872200 & -0.88613700 & 0.64009100 \\ \mathrm{H} & -7.64874600 & 0.88596000 & 0.64015700 \\ \mathrm{C} & -9.38764100 & -0.00008200 & -0.25027300 \\ \mathrm{H} & -9.66796500 & -0.89117700 & -0.82564800 \\ \mathrm{H} & -9.66799300 & 0.89103200 & -0.82560600 \\ \mathrm{O} & -10.06940600 & -0.00012300 & 1.01791500 \\ \mathrm{C} & -11.42857700 & -0.00010300 & 1.10681600 \\ \mathrm{C} & -12.22853400 & -0.00002700 & -0.18040000 \\ \mathrm{H} & -12.00534300 & 0.88362200 & -0.78721500 \\ \mathrm{H} & -12.00516600 & -0.88344000 & -0.78748600 \\ \mathrm{H} & -13.28667900 & -0.00016100 & 0.07811600 \\ \mathrm{O} & -11.93497000 & -0.00013000 & 2.20517200 \\ \mathrm{H} & 7.64870500 & 0.88587000 & -0.64026900 \\ \mathrm{C} & 9.38764100 & 0.00005900 & 0.25030300 \\ \mathrm{H} & 9.66792100 & 0.89127600 & 0.82550700 \\ \mathrm{H} & 9.66801600 & -0.89092500 & 0.82582000 \\ \mathrm{O} & 10.06943000 & -0.00013200 & -1.01787200 \\ \mathrm{C} & 11.42860100 & -0.00017800 & -1.10672900 \\ \mathrm{C} & 12.22850700 & -0.00015200 & 0.18051500 \\ \mathrm{H} & 12.00510200 & 0.88327300 & 0.78756300 \\ \mathrm{H} & 12.00529700 & -0.88379100 & 0.78733800 \\ \mathrm{H} & 13.28666300 & -0.00002500 & -0.07794600 \\ \mathrm{O} & 11.93503400 & -0.00028800 & -2.20506800 \\ & & & \end{array}$

PDI-Br ground state optimized coordinates calculated at B3LYP/6-31+G(d,p) level of theory

$\begin{array}{lrrr}\mathrm{C} & 1.47979200 & 2.11278300 & 0.09945400 \\ \mathrm{C} & 0.64737100 & 0.98131300 & 0.13384700 \\ \mathrm{C} & -0.82717900 & 0.98244900 & 0.08576700 \\ \mathrm{C} & -1.60753900 & 2.09411600 & 0.41394500 \\ \mathrm{C} & 1.30911900 & -0.29843100 & 0.21195300 \\ \mathrm{C} & -1.50523600 & -0.22352000 & -0.29059600 \\ \mathrm{C} & -0.81571200 & -1.46807600 & -0.39813600 \\ \mathrm{C} & 0.59413600 & -1.52155900 & 0.00229400 \\ \mathrm{C} & -1.51344500 & -2.59259200 & -0.84665000 \\ \mathrm{C} & -2.88236400 & -2.54080300 & -1.13382900 \\ \mathrm{C} & -2.91894200 & -0.20010200 & -0.49942200 \\ \mathrm{C} & -3.65439700 & 0.98437900 & -0.24740300 \\ \mathrm{C} & -3.00093700 & 2.09602600 & 0.25368600 \\ \mathrm{H} & -3.58099000 & 2.97811500 & 0.50270100 \\ \mathrm{H} & -3.41072600 & -3.42029400 & -1.48549600 \\ \mathrm{C} & 2.86363200 & 2.03306600 & 0.33295700 \\ \mathrm{C} & 3.46826700 & 0.81896300 & 0.57677800 \\ \mathrm{C} & 2.70968800 & -0.37171200 & 0.48156200 \\ \mathrm{H} & 3.47018500 & 2.93073000 & 0.33534800 \\ \mathrm{C} & 2.62420100 & -2.79276400 & 0.49612600 \\ \mathrm{C} & 1.26614400 & -2.73593900 & 0.16896300 \\ \mathrm{H} & 3.12738400 & -3.74450600 & 0.62731700\end{array}$




\begin{tabular}{|c|c|c|c|}
\hline & -5.12065400 & 1.02879000 & -0.46625600 \\
\hline & 3.35330600 & -1.62265700 & 0.63628700 \\
\hline & 4.80315900 & -1.69816900 & 0.93916600 \\
\hline & 4.92441900 & 0.78077200 & 0.86252400 \\
\hline & 5.60434800 & 1.79789100 & 0.93359100 \\
\hline & 5.38688600 & -2.76564900 & 1.08777300 \\
\hline & -5.78819400 & 2.03169500 & -0.23958300 \\
\hline & -3.59209700 & -1.36555900 & -0.94132300 \\
\hline & -5.05371700 & -1.34689900 & -1.19221600 \\
\hline & -5.66326800 & -2.33402600 & -1.58904200 \\
\hline & -5.72582900 & -0.13799400 & -0.95641000 \\
\hline & 5.49669500 & -0.48295300 & 1.05507700 \\
\hline B & 0.90274200 & 3.88708800 & -0.33509700 \\
\hline & -7.17866800 & -0.10031700 & -1.20938200 \\
\hline & -7.37408500 & -0.79626300 & -2.02635800 \\
\hline & -7.41756400 & 0.91549500 & -1.52792500 \\
\hline & -7.99242600 & -0.48161000 & 0.03440700 \\
\hline & -7.71727800 & -1.49109600 & 0.35772300 \\
\hline & -7.76188600 & 0.21204700 & 0.84965100 \\
\hline & -9.48421600 & -0.43386700 & -0.26131900 \\
\hline & -9.80744500 & 0.56412100 & -0.57562600 \\
\hline & -9.77022500 & -1.14267100 & -1.04545200 \\
\hline & -10.17687100 & -0.78462100 & 0.95950000 \\
\hline & -11.52992300 & -0.79598700 & 0.90085300 \\
\hline & -12.13031100 & -1.15913400 & 2.23658700 \\
\hline & -11.72816900 & -2.11456100 & 2.58602600 \\
\hline & -11.86606000 & -0.40066300 & 2.98028900 \\
\hline & -13.21419600 & -1.22008200 & 2.14161900 \\
\hline & -12.15569200 & -0.53779500 & -0.10704400 \\
\hline & 6.93701300 & -0.54162200 & 1.36998000 \\
\hline & 7.17052200 & 0.35649800 & 1.94380600 \\
\hline & 7.08715100 & -1.42417500 & 1.99331200 \\
\hline & 7.80325700 & -0.61782800 & 0.10588200 \\
\hline & 7.53386200 & -1.50933800 & -0.47025300 \\
\hline & 7.61757000 & 0.26137800 & -0.51976900 \\
\hline & 9.27992900 & -0.67935600 & 0.46791500 \\
\hline & 9.51510300 & -1.55619500 & 1.08029000 \\
\hline & 9.60515200 & 0.21096900 & 1.01634100 \\
\hline & 10.02408500 & -0.76223300 & -0.76968100 \\
\hline & 11.37171900 & -0.84008700 & -0.65581100 \\
\hline & 12.02872200 & -0.93121600 & -2.01089500 \\
\hline & 13.11086800 & -0.96346500 & -1.88686100 \\
\hline & 11.68684100 & -1.83126700 & -2.53128100 \\
\hline & 11.74685600 & -0.07080000 & -2.62514500 \\
\hline & 11.95133800 & -0.83796200 & 0.41095500 \\
\hline & -1.14421300 & 2.98447000 & 0.80892500 \\
\hline & 0.72953500 & -3.66964200 & 0.05263400 \\
\hline & -0.99792400 & -3.53577200 & -0.98204000 \\
\hline
\end{tabular}

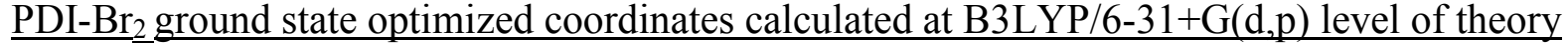

$\begin{array}{lrrc}\mathrm{C} & 1.56788500 & 2.49806600 & -0.23696800 \\ \mathrm{C} & 0.73097200 & 1.39729500 & 0.00316000 \\ \mathrm{C} & -0.73946700 & 1.40373900 & 0.02065000 \\ \mathrm{C} & -1.49282600 & 2.55318900 & 0.27297800 \\ \mathrm{C} & 1.37348800 & 0.14257000 & 0.27987800 \\ \mathrm{C} & -1.43464000 & 0.17210800 & -0.21050600 \\ \mathrm{C} & -0.74029200 & -1.08538900 & -0.23231500 \\ \mathrm{C} & 0.63819300 & -1.08748800 & 0.28031000 \\ \mathrm{C} & -1.45051700 & -2.19225700 & -0.72234400 \\ \mathrm{C} & -2.83079900 & -2.13664600 & -0.97865900 \\ \mathrm{C} & -2.85000700 & 0.20600900 & -0.38181900\end{array}$




\begin{tabular}{|c|c|c|c|}
\hline & -3.56957700 & 1.40869200 & -0.18659200 \\
\hline & -2.89124400 & 2.55566200 & 0.18866400 \\
\hline & -3.45523600 & 3.46088700 & 0.38545200 \\
\hline & -3.35927200 & -3.01343600 & -1.33303300 \\
\hline & 2.95317600 & 2.44314900 & -0.00891300 \\
\hline & 3.53799600 & 1.28706900 & 0.46635800 \\
\hline & 2.76258400 & 0.11132300 & 0.60092400 \\
\hline & 3.57301200 & 3.31539800 & -0.17835200 \\
\hline & 2.60356200 & -2.23027000 & 1.18384200 \\
\hline & 1.25898200 & -2.23072400 & 0.79037200 \\
\hline & 3.06589800 & -3.13113200 & 1.57236100 \\
\hline & -5.04305200 & 1.44398800 & -0.35317600 \\
\hline & 3.37067300 & -1.08608600 & 1.04688700 \\
\hline & 4.81251400 & -1.11877600 & 1.39309400 \\
\hline 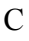 & 4.99227100 & 1.27921300 & 0.76583500 \\
\hline & 5.69613300 & 2.27102400 & 0.61651200 \\
\hline 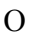 & 5.36644700 & -2.13436100 & 1.79680800 \\
\hline 0 & -5.70124800 & 2.45772200 & -0.15239600 \\
\hline & -3.53773500 & -0.97330200 & -0.75306700 \\
\hline & -5.00655100 & -0.96298000 & -0.97026300 \\
\hline & -5.62322200 & -1.96428900 & -1.31439600 \\
\hline N & -5.66791100 & 0.25478100 & -0.76242300 \\
\hline J & 5.53358400 & 0.07645400 & 1.23898100 \\
\hline$B_{1}$ & 0.96979200 & 4.16391200 & -0.96525000 \\
\hline$B$ & -0.64851000 & -3.86899100 & -1.17967200 \\
\hline & -7.12896000 & 0.28376900 & -0.96768300 \\
\hline H & -7.35051500 & -0.43651500 & -1.75652700 \\
\hline $\mathrm{H}$ & -7.37951100 & 1.28924300 & -1.30875400 \\
\hline & -7.89876400 & -0.06120300 & 0.31399300 \\
\hline & -7.60930400 & -1.05957400 & 0.65854800 \\
\hline & -7.64332200 & 0.65751500 & 1.09970200 \\
\hline & -9.39987600 & -0.02797300 & 0.06709100 \\
\hline H & -9.74011300 & 0.96130500 & -0.25659100 \\
\hline & -9.70637600 & -0.75443000 & -0.69279700 \\
\hline ] & -10.04877700 & -0.35855000 & 1.31678600 \\
\hline & -11.40320500 & -0.38422000 & 1.30180000 \\
\hline & -11.95680600 & -0.73332000 & 2.66113900 \\
\hline $\mathrm{H}$ & -11.55266000 & -1.69240900 & 2.99845400 \\
\hline & -11.65594700 & 0.02414100 & 3.39164500 \\
\hline & -13.04401200 & -0.78361900 & 2.60690900 \\
\hline & -12.06282900 & -0.14953200 & 0.30999300 \\
\hline & 6.96989700 & 0.05791200 & 1.57650400 \\
\hline$H$ & 7.22301000 & 1.05728300 & 1.93336400 \\
\hline & 7.09312300 & -0.66259800 & 2.38632100 \\
\hline & 7.84186800 & -0.32425900 & 0.37320600 \\
\hline & 7.54848900 & -1.31363900 & 0.00683200 \\
\hline & 7.68839400 & 0.39773500 & -0.43565700 \\
\hline & 9.31338600 & -0.34441500 & 0.75999700 \\
\hline & 9.52076500 & -1.07986700 & 1.54430100 \\
\hline & 9.65664500 & 0.63274900 & 1.11590300 \\
\hline & 10.06530600 & -0.69960500 & -0.42344400 \\
\hline & 11.41003400 & -0.77830900 & -0.27914400 \\
\hline & 12.07754800 & -1.14362700 & -1.58184200 \\
\hline & 13.15061700 & -1.24621700 & -1.42221100 \\
\hline & 11.66370500 & -2.07906600 & -1.96982300 \\
\hline & 11.88630000 & -0.36628200 & -2.32829900 \\
\hline & 11.98019300 & -0.57220900 & 0.77268700 \\
\hline & -0.99580700 & 3.47231200 & 0.54551900 \\
\hline & 0.69723800 & -3.14732900 & 0.89234100 \\
\hline
\end{tabular}

$\underline{\mathrm{PDI}_{-} \mathrm{Br}_{3}}$ ground state optimized coordinates calculated at B3LYP/6-31+G(d,p) level of theory 


\begin{tabular}{|c|c|c|c|}
\hline $\mathrm{C}$ & -1.53270000 & 2.84331400 & -0.00827400 \\
\hline $\mathrm{C}$ & -0.69206900 & 1.73135100 & -0.16935100 \\
\hline $\mathrm{C}$ & 0.77980800 & 1.74019800 & -0.21597700 \\
\hline $\mathrm{C}$ & 1.55047500 & 2.84494900 & -0.58832300 \\
\hline $\mathrm{C}$ & -1.33681800 & 0.45504500 & -0.32376100 \\
\hline $\mathrm{C}$ & 1.46424000 & 0.53891700 & 0.15015600 \\
\hline $\mathrm{C}$ & 0.75138600 & -0.68510800 & 0.34284000 \\
\hline $\mathrm{C}$ & -0.57949400 & -0.76140200 & -0.26147400 \\
\hline $\mathrm{C}$ & 1.38052800 & -1.67579500 & 1.10687000 \\
\hline $\mathrm{C}$ & 2.75561000 & -1.61736600 & 1.39392700 \\
\hline $\mathrm{C}$ & 2.86858900 & 0.57501500 & 0.37226700 \\
\hline $\mathrm{C}$ & 3.60914800 & 1.72819300 & 0.02598200 \\
\hline $\mathrm{C}$ & 2.95080700 & 2.82829100 & -0.50101700 \\
\hline $\mathrm{H}$ & 3.53137500 & 3.69394600 & -0.80080200 \\
\hline $\mathrm{H}$ & 3.23826500 & -2.41543600 & 1.94580200 \\
\hline $\mathrm{C}$ & -2.92662600 & 2.75684100 & -0.16641200 \\
\hline $\mathrm{C}$ & -3.51716700 & 1.55819000 & -0.51563900 \\
\hline $\mathrm{C}$ & -2.72959400 & 0.38889400 & -0.60021100 \\
\hline $\mathrm{H}$ & -3.54846700 & 3.63595700 & -0.04731100 \\
\hline $\mathrm{C}$ & -2.52171400 & -1.95463700 & -1.16196900 \\
\hline $\mathrm{C}$ & -1.15681600 & -1.90352000 & -0.83320700 \\
\hline $\mathrm{H}$ & -2.96143700 & -2.86351300 & -1.55557700 \\
\hline $\mathrm{C}$ & 5.07915400 & 1.75940900 & 0.21950200 \\
\hline $\mathrm{C}$ & -3.31918600 & -0.84241800 & -0.97284000 \\
\hline $\mathrm{C}$ & -4.77226600 & -0.92403900 & -1.26488600 \\
\hline $\mathrm{C}$ & -4.98132400 & 1.50238400 & -0.75441700 \\
\hline $\mathrm{O}$ & -5.70205000 & 2.48506800 & -0.63316300 \\
\hline $\mathrm{O}$ & -5.31301800 & -1.96908000 & -1.60402200 \\
\hline $\mathrm{O}$ & 5.76552100 & 2.71634700 & -0.11791000 \\
\hline $\mathrm{C}$ & 3.51003500 & -0.54493800 & 0.95560600 \\
\hline $\mathrm{C}$ & 4.97276600 & -0.52403500 & 1.20883200 \\
\hline $\mathrm{O}$ & 5.55990000 & -1.46383700 & 1.73109800 \\
\hline $\mathrm{N}$ & 5.66586900 & 0.63195300 & 0.81946300 \\
\hline $\mathrm{N}$ & -5.51333500 & 0.26103400 & -1.13440700 \\
\hline $\mathrm{Br}$ & -0.91381700 & 4.57609200 & 0.51063200 \\
\hline $\mathrm{Br}$ & 0.39379900 & -3.05633000 & 1.96508500 \\
\hline $\mathrm{Br}$ & -0.10934400 & -3.39593000 & -1.37404300 \\
\hline $\mathrm{C}$ & 7.12212900 & 0.66423600 & 1.05733400 \\
\hline $\mathrm{H}$ & 7.30616800 & 0.08703200 & 1.96475500 \\
\hline $\mathrm{H}$ & 7.39032900 & 1.70795200 & 1.22673900 \\
\hline $\mathrm{C}$ & 7.91260700 & 0.08478100 & -0.12300700 \\
\hline $\mathrm{H}$ & 7.60552300 & -0.95196800 & -0.29653600 \\
\hline $\mathrm{H}$ & 7.69487200 & 0.66019300 & -1.02901300 \\
\hline $\mathrm{C}$ & 9.40775000 & 0.12693400 & 0.15649800 \\
\hline $\mathrm{H}$ & 9.76835700 & 1.15020100 & 0.30525700 \\
\hline $\mathrm{H}$ & 9.67506000 & -0.45638400 & 1.04386000 \\
\hline $\mathrm{O}$ & 10.07672900 & -0.44046000 & -0.99357400 \\
\hline $\mathrm{C}$ & 11.42836100 & -0.50652500 & -0.93020700 \\
\hline $\mathrm{C}$ & 12.00319400 & -1.11956800 & -2.18316400 \\
\hline $\mathrm{H}$ & 11.61403300 & -2.13374000 & -2.31587800 \\
\hline $\mathrm{H}$ & 11.70284700 & -0.53637000 & -3.05890400 \\
\hline $\mathrm{H}$ & 13.08994800 & -1.14583100 & -2.10736200 \\
\hline $\mathrm{O}$ & 12.07027100 & -0.11710000 & 0.02381800 \\
\hline $\mathrm{C}$ & -6.96233200 & 0.19427800 & -1.40834600 \\
\hline $\mathrm{H}$ & -7.25287500 & 1.16704100 & -1.80754600 \\
\hline $\mathrm{H}$ & -7.10307200 & -0.57194300 & -2.17191900 \\
\hline $\mathrm{C}$ & -7.77234800 & -0.13867100 & -0.14859400 \\
\hline $\mathrm{H}$ & -7.44389500 & -1.10213500 & 0.25530500 \\
\hline $\mathrm{H}$ & -7.59722900 & 0.62750500 & 0.61395700 \\
\hline $\mathrm{C}$ & -9.25910100 & -0.20368100 & -0.46634900 \\
\hline $\mathrm{H}$ & -9.48757200 & -0.98279300 & -1.20117400 \\
\hline $\mathrm{H}$ & -9.63612400 & 0.74755100 & -0.85653900 \\
\hline
\end{tabular}




$\begin{array}{lccc}\mathrm{O} & -9.94998800 & -0.50831700 & 0.76698000 \\ \mathrm{C} & -11.29932200 & -0.60618600 & 0.69023500 \\ \mathrm{C} & -11.90053100 & -0.92004800 & 2.03774100 \\ \mathrm{H} & -12.98255900 & -1.00466500 & 1.94022800 \\ \mathrm{H} & -11.48539900 & -1.85488900 & 2.42636000 \\ \mathrm{H} & -11.65116500 & -0.12917300 & 2.75183400 \\ \mathrm{O} & -11.91990700 & -0.45452100 & -0.34203400 \\ \mathrm{H} & 1.07022800 & 3.74194200 & -0.95129300\end{array}$

$\underline{\mathrm{PDI}_{-B r}} 4$ ground state optimized coordinates calculated at B3LYP/6-31+G(d,p) level of theory

2.474463

$0.72926400 \quad 1.41310400-0.07388800$

$\begin{array}{llll}\mathrm{C} & 1.53186800 & 2.47452100 & -0.51186400\end{array}$

$\begin{array}{llll}\mathrm{C} & -1.39626100 & 0.18700900 & -0.24252500\end{array}$

C $\quad 1.39626300 \quad 0.18706800 \quad 0.24251000$

C $\quad 0.66211300 \quad-1.03925400 \quad 0.31494500$

C $\quad-0.66206000-1.03928400-0.31494400$

C $\quad 1.26967500 \quad-2.10007500 \quad 0.99919800$

$\begin{array}{llll}\mathrm{C} & 2.63527800 & -2.07271900 & 1.33532900\end{array}$

$\begin{array}{llll}\mathrm{C} & 2.79258200 & 0.18827200 & 0.48847300\end{array}$

$\begin{array}{llll}\mathrm{C} & 3.54835200 & 1.34892400 & 0.20409200\end{array}$

$\begin{array}{llll}\mathrm{C} & 2.93073800 & 2.44735300 & -0.36700200\end{array}$

$\mathrm{H} \quad 3.53330400 \quad 3.29207500 \quad-0.67951000$

$\begin{array}{lll}3.09711700 & -2.91803700 & 1.83180600\end{array}$

$\begin{array}{lll}-2.93083200 & 2.44723700 & 0.36695700\end{array}$

$\begin{array}{lll}-3.54839600 & 1.34878100 & -0.20414000\end{array}$

$\begin{array}{lll}-2.79257700 & 0.18815600 & -0.48850300\end{array}$

$\begin{array}{lll}-3.53343500 & 3.29193400 & 0.67945800\end{array}$

$-2.63517600-2.07283800 \quad-1.33533200$

$-1.26957400 \quad-2.10013500 \quad-0.99919200$

$-3.09697700 \quad-2.91818200 \quad-1.83179900$

$\begin{array}{lll}5.01479300 & 1.35663100 & 0.43225500\end{array}$

$-3.40827500-0.97218700-1.01159800$

$-4.86433200 \quad-0.97676100-1.29939300$

$\begin{array}{lll}-5.01483400 & 1.35642900 & -0.43232400\end{array}$

$\begin{array}{lll}-5.71626500 & 2.32052800 & -0.15359400\end{array}$

$-5.43373000 \quad-1.94853200-1.77961600$

$\begin{array}{lll}5.71617900 & 2.32076000 & 0.15351400\end{array}$

$\begin{array}{lll}3.40833100 & -0.97204200 & 1.01157200\end{array}$

$\begin{array}{lll}4.86439000 & -0.97655200 & 1.29935600\end{array}$

$\begin{array}{lll}5.43383300 & -1.94829000 & 1.77959500\end{array}$

$\begin{array}{llll}5.57393400 & 0.19640600 & 0.99286100\end{array}$

$\begin{array}{llll}-5.57392300 & 0.19617700 & -0.99292500\end{array}$

$\begin{array}{lll}-0.80942800 & 3.93236900 & 1.49443800\end{array}$

$\begin{array}{llll}0.80926400 & 3.93241200 & -1.49444500\end{array}$

$\begin{array}{lll}0.25755800 & -3.55683100 & 1.68162700\end{array}$

$-0.25740600 \quad-3.55688200 \quad-1.68156800$

$\begin{array}{lll}7.02590900 & 0.20143000 & 1.26027300\end{array}$

$\begin{array}{lll}7.18747000 & -0.43511900 & 2.13134000\end{array}$

$\begin{array}{llll}7.29846900 & 1.22946700 & 1.50288900\end{array}$

$\begin{array}{lll}7.83463100 & -0.30634800 & 0.05948200\end{array}$

$\begin{array}{lll}7.52445600 & -1.32750800 & -0.18627500\end{array}$

$\begin{array}{llll}7.63766600 & 0.32800400 & -0.81108600\end{array}$

$\begin{array}{lll}9.32466500 & -0.29278500 & 0.36794700\end{array}$

$\begin{array}{llll}9.68761900 & 0.71590900 & 0.59163500\end{array}$

$\begin{array}{llll}9.57286500 & -0.93596800 & 1.21877700\end{array}$

$10.01096700 \quad-0.78577900 \quad-0.80566200$

$11.36177100 \quad-0.85637600 \quad-0.72580300$

$11.95590300 \quad-1.39219600 \quad-2.00478700$

$\begin{array}{lll}11.59670300 & -2.41048600 & -2.18378700\end{array}$ 


$\begin{array}{lccc}\mathrm{H} & 11.63987700 & -0.77933800 & -2.85411400 \\ \mathrm{H} & 13.04280900 & -1.39231300 & -1.92658700 \\ \mathrm{O} & 11.98828100 & -0.52810600 & 0.26080700 \\ \mathrm{C} & -7.02589600 & 0.20114700 & -1.26034900 \\ \mathrm{H} & -7.29847900 & 1.22915500 & -1.50306300 \\ \mathrm{H} & -7.18743200 & -0.43548600 & -2.13135900 \\ \mathrm{C} & -7.83462000 & -0.30654700 & -0.05952300 \\ \mathrm{H} & -7.52441300 & -1.32767500 & 0.18632900 \\ \mathrm{H} & -7.63769000 & 0.32789000 & 0.81099100 \\ \mathrm{C} & -9.32464900 & -0.29306400 & -0.36801100 \\ \mathrm{H} & -9.57278700 & -0.93628400 & -1.21883600 \\ \mathrm{H} & -9.68766700 & 0.71559700 & -0.59172700 \\ \mathrm{O} & -10.01094500 & -0.78610100 & 0.80558400 \\ \mathrm{C} & -11.36178700 & -0.85619300 & 0.72591400 \\ \mathrm{C} & -11.95591800 & -1.39209800 & 2.00486400 \\ \mathrm{H} & -13.04284100 & -1.39163000 & 1.92690900 \\ \mathrm{H} & -11.59721700 & -2.41064300 & 2.18342900 \\ \mathrm{H} & -11.63936000 & -0.77970200 & 2.85432400 \\ \mathrm{O} & -11.98832600 & -0.52748000 & -0.26053000\end{array}$

\section{References:}

1. S. W. Eaton, L. E. Shoer, S. D. Karlen, S. M. Dyar, E. A. Margulies, B. S. Veldkamp, C. Ramanan, D. A. Hartzler, S. Savikhin, T. J. Marks and M. R. Wasielewski, J. Am. Chem. Soc., 2013, 135, 14701-14712.

2. T. Hinoue, Y. Shigenoi, M. Sugino, Y. Mizobe, I. Hisaki, M. Miyata and N. Tohnai, Chem. Eur. J., 2012, 18, 4634-4643.

3. D. F. Eaton, Pure Appl. Chem., 1988, 60, 1107-1114.

4. M. Cölle, J. Gmeiner, W. Milius, H. Hillebrecht and W. Brütting, Adv. Funct. Mater., 2003, 13, 108-112.

5. J. J. Snellenburg, S. P. Laptenok, R. Seger, K. M. Mullen and I. H. M. van Stokkum, J. Stat. Soft., 2012, 49, 1-22.

6. W. E. Ford and P. V. Kamat, J. Phys. Chem., 1987, 91, 6373-6380.

7. K. Sandros, Acta Chem. Scand., 1964, 18, 2355-2374.

8. M. J. Frisch, G. W. Trucks, H. B. Schlegel, G. E. Scuseria, M. A. Rob, J. R. Cheeseman, J. A. Montgomery Jr., T. K. Vreven, K. N., J. C. Burant, J. M. Millam, S. S. Iyengar, J. Tomasi, V. Barone, B. Mennucci, M. Cossi, G. Scalmani, N. Rega, G. A. Petersson, H. Nakatsuji, M. Hada, M. Ehara, K. Toyota, R. Fukuda, J. Hasegawa, M. Ishida, Y. Nakajima, O. Honda, H. Kitao, M. Nakai, X. Klene, J. E. Li, H. P. Knox, J. B. Hratchian, T. Cross, V. Bakken, C. Adamo, J. Jaramillo, R. Gomperts, R. E. Stratmann, O. Yazyev, A. J. Austin, R. Cammi, C. Pomelli, J. W. Ochterski, P. Y. Ayala, K. Morokuma, G. A. Voth, P. Salvador, J. J. Dannenberg, V. G. Zakrzewski, S. Dapprich, A. D. Daniels, M. C. Strain, O. Farkas, D. K. Malick, A. D. Rabuck, K. Raghavachari, J. B. Foresman, J. V. Ortiz, Q. Cui, A. G. Baboul, S. Clifford, J. Cioslowski, B. B. Stefanov, G. Liu, A. Liashenko, P. Piskorz, I. Komaromi, R. L. Martin, D. J. Fox, T. Keith, M. A. Al-Laham, C. Y. Peng, A. Nanayakkara, M. Challacombe, P. M. W. Gill, B. Johnson, W. Chen, M. W. Wong, C. Gonzalez and J. A. Pople, (Gaussian, Inc., Wallingford, CT, 2003). 